\title{
Stability analysis of nontrivial stationary solution and constant equilibrium point of reaction-diffusion neural networks with time delays under Dirichlet zero boundary value
}

\author{
Ruofeng $\operatorname{Rao}^{\mathrm{a}, *}$ \\ ${ }^{a}$ Department of Mathematics, Chengdu Normal University, Chengdu, 611130, China
}

\begin{abstract}
In this paper, Lyapunov-Razumikhin technique, design of state-dependent switching laws, a fixed point theorem and variational methods are employed to derive the existence and the unique existence results of (globally) exponentially stable positive stationary solution of delayed reaction-diffusion cell neural networks under Dirichlet zero boundary value, including the global stability criteria in the classical meaning. Next, sufficient conditions are proposed to guarantee the global stability invariance of ordinary differential systems under the influence of diffusions. New theorems show that the diffusion is a double-edged sword in judging the stability of diffusion systems. Besides, an example is constructed to illuminate that any non-zero constant equilibrium point must be not in the phase plane of dynamic system under Dirichlet zero boundary value, or it must lead to a contradiction. Next, under Lipschitz assumptions on active function, another example is designed to prove that the small diffusion effect will cause the essential change of the phase plane structure of the dynamic behavior of the delayed neural networks via a Saddle point theorem. Finally, a numerical example illustrates the feasibility of the proposed methods.
\end{abstract}

Keywords: reaction-diffusion; cellular neural networks; exponential stability; stationary solutions ; Saddle point theorem

\section{Introduction}

Firstly, we recalled the reason why we need to study the stability of reaction-diffusion neural networks system.

In 1988, inspired by cellular automata, Chua and Yang proposed a new neural network based on Hopfield network, i.e. cellular neural network $(\mathrm{CNN})$, which is formed by a number of cells with the same structure after a well-organized combination ([22,23]). Each neuron in the network will automatically choose to connect with the nearest neuron. Because of its local connectivity, $\mathrm{CNN}$ is especially suitable for ultra large scale integrated circuit implementation. The characteristics of the above cellular neural network make it widely used in pattern recognition, image processing, signal processing and other fields. The main function of cellular neural network is to transform an input image

\footnotetext{
*The work was jointly supported by the Application basic research project of science and Technology Department of Sichuan Province (2020YJ0434) and the Major Scientific research projects of Chengdu Normal University in 2019 (CS19ZDZ01).

Email address: ruof engrao@163.com (Ruofeng Rao )
} 
into a corresponding output image. For example, the existing target motion direction detection, edge detection, and connected slice detection all use this function. In order to achieve these functions, the cellular neural network must be completely stable, that is, all output trajectories must converge to a stable equilibrium point. So the stability of cellular neural network has become a hot topic ([24-26]). As we all know, time delay may destroy the stability of the system and lead to oscillation, bifurcation, chaos and other phenomena, thus changing the characteristics of the system. In cellular neural networks, time delay is inevitable. For example, there are cell delay, transmission delay and synapse delay in biological neural network ([27]). As pointed out in [28] that many pattern formation and wave propagation phenomena that appear in nature can be described by systems of coupled nonlinear differential equations, generally known as reaction-diffusion equations. These wave propagation phenomena are exhibited by systems belonging to very different scientific disciplines. Besides, the interactions arising from the space-distributed structure of the multilayer cellular neural networks can be seen as diffusion phenomenon([3, 29]). Thereby, the reaction-diffusion effects cannot be neglected in both biological and man-made neural networks, especially when electrons are moving in non-even electromagnetic field. Moreover, although the diffusion coefficients may be very small, the topological structure of the phase plane of the dynamic behavior of the following reaction-diffusion system (1.1) is likely to change substantially from a constant equilibrium point of the following system (1.3) to multiple stationary solutions of the reaction-diffusion system (1.1). Therefore, many global stability results of delayed neural networks in the form of ordinary differential equations may only be locally asymptotical stability criteria in real engineering. Unfortunately, such an example has not been constructed for the time being. But that doesn't mean there are no such examples, which may become an open problem hereafter. On the other hand, fortunately, this paper has proposed the conditions guaranteeing the global stability invariance of ordinary differential systems under the influence of diffusions in the meaning of Definition 1 (see Corollary 3.4).

Next, we shall point out the fact that the stability results in previous literature involved to delayed reactiondiffusion neural networks make it unnecessary to study the reaction-diffusion system (partial differential equations model), but only its corresponding ordinary differential equations model. What's the problem?

For a long time, the stability of the reaction diffusion neural networks was investigated in many literatures[1-10], in which the stability of the constant equilibrium point was studied. For example, in [1], the following cellular neural networks with time-varying delays and reaction-diffusion terms was considered,

$$
\begin{aligned}
\frac{\partial y(t, x)}{\partial t}= & \sum_{q=1}^{m} \frac{\partial y(t, x)}{\partial x_{q}}\left(D_{q} \frac{\partial y(t, x)}{\partial x_{q}}\right)-C y(t, x)+A g(y(t, x)) \\
& +B g(y(t-\tau(t), x))+J,(t, x) \in \mathbb{R}_{+} \times \Omega,
\end{aligned}
$$

Next, the authors of [1] defined the equilibrium point of the time-delayed reaction-diffusion system (1.1) as the constant vector $y^{*}$ satisfying

$$
C y^{*}=A g\left(y^{*}\right)+B g\left(y^{*}\right)+J
$$


Here, we have to say, the equilibrium point $y^{*}$ is also the equilibrium point of the following ordinary differential equations corresponding to the time-delayed partial differential equations (1.1),

$$
\frac{d x(t)}{d t}=-C x(t)+A g(x(t))+B g(x(t-\tau(t)))+J, t \in \mathbb{R}_{+}
$$

Due to the Poincare inequality, we see, the diffusion items actually promote the stability of the reaction diffusion system (1.1). That is, we only need to study the ordinary differential equations (1.3) because the stability criteria of the ordinary differential equations (1.3) must make the system (1.1) stable. In other words, the stability of the reaction-diffusion model does not need to be studied because it is included in the stability of its corresponding ordinary differential equations model.

So we need to ask where the problem is? The answer lies in the fact pointed out in this paper. In Theorem 3.3 and Corollary 3.4, the constant equilibrium point $u^{*}$ may become another $u^{*}(x)$, where $u^{*}(x) \neq u^{*}$ in common cases under Dirichlet zero boundary value. Only the zero solution $u^{*}=0$ might become one of the stationary solutions of reaction-diffusion system. And so the uniqueness existence conditions of the stationary solution $u^{*}(x)$ (see Corollary 3.4) illuminate the inconvenient and difficulties due to the inevitable diffusions in practical engineering, which make it more difficult to judge the stability than ordinary differential system. Usually, the positive stationary solution $u^{*}(x)>0$ implies more realistic meanings in in neural networks and other systems, such as the financial systems ([11,12,30]). And so Theorem 3.1 and Theorem 3.2 have proposed the existence and the unique existence of the (globally) exponentially stable positive stationary solution in this paper.

Motivated by some methods of [1-31], we investigate the stability of the nontrivial stationary solution of switched reaction-diffusion neural networks with time delays. This paper has the following innovations:

It is the first paper to study and obtain the existence theorem and unique existence theorem (see Theorem 3.1 and Theorem 3.2) of (globally) asymptotically stable nontrivial stationary solution of reaction-diffusion neural networks with time delays under Dirichlet zero boundary value via the comprehensive applications of Lyapunov-Razumikhin technique, design of state-dependent switching laws, a fixed point theorem, variational methods, and construction of compact operators on a convex set. Moreover, such new theorems illuminate originally that the diffusion phenomena is the double-edged sword in judging the stability of delayed reaction-diffusion systems.

$\star$ It is the first paper to propose the conditions guaranteeing the global stability invariance of delayed ordinary differential systems under the influence of diffusions in the meaning of Definition 1 (see Theorem 3.3 and Corollary $3.4)$.

$\star$ It is the first paper to design the contradiction results to show that any non-zero constant function must not be a solution of any reaction diffusion neural networks under Dirichlet zero boundary value.

- It is also the first time to study how the tiny diffusion causes the essential change of the phase plane structure of the dynamic behavior of the delayed neural networks under Lipschitz assumptions on activate functions or signal functions. 


\section{Notations :}

Throughout this paper, we denote by $I$ the identity matrix with an appropriate dimension, and assume that $D_{\sigma}$ is a positive definite diagonal matrix for any given $\sigma \in\{1,2, \cdots, N\}$. Besides, $\operatorname{diag}(\cdots)$ stands for a diagonal matrix. $\mathcal{A}>0(\mathcal{A} \geqslant 0)$ means that $\mathcal{A}$ is a real symmetric positive (semi-positive) definite matrix. And denote $|\mathcal{A l}|=\left(\left|a_{i j}\right|\right)_{n \times n}$ for any matrix $\mathcal{A}=\left(a_{i j}\right)_{n \times n}$. In addition, for any $v=\left(v_{1}, v_{2}, \cdots, v_{n}\right)^{T}, u=\left(u_{1}, u_{2}, \cdots, u_{n}\right)^{T} \in \mathbb{R}^{n}$, we denote $|v|=\left(\left|v_{1}\right|,\left|v_{2}\right|, \cdots,\left|v_{n}\right|\right)^{T}$, and $v \leqslant u$ means that $v_{i} \leqslant u_{i}$ for all $i=1,2, \cdots, n$. Denote by $\Omega_{\sigma}$ an open bounded domain in $R^{n}$ with the smooth boundary $\partial \Omega_{\sigma}$ for any given $\sigma \in\{1,2, \cdots, N\}$. For convenience, we denote by $\lambda_{\sigma 1}>0$ the first positive eigenvalue of the Laplace operator $-\Delta$ on the Sobolev space $W_{0}^{1,2}\left(\Omega_{\sigma}\right)$.

\section{System descriptions}

Consider the following switched neural networks with time-varying delays and reaction-diffusion terms

$$
\left\{\begin{array}{l}
\frac{\partial y(t, x)}{\partial t}=D_{\sigma} \Delta y(t, x)-C_{\sigma} y(t, x)+A_{\sigma} g(y(t, x))+B_{\sigma} g(y(t-\tau(t), x))+J_{\sigma}, \quad(t, x) \in \mathbb{R}_{+} \times \Omega_{\sigma}, \\
y_{i}(t, x)=0, t \geqslant 0, x \in \partial \Omega_{\sigma}, i=1,2, \cdots, n,
\end{array}\right.
$$

where $\Omega_{\sigma} \subset \mathbb{R}^{n}$ is a bounded domain with smooth boundary $\partial \Omega_{\sigma}$, the state variable $y(t, x)=\left(y_{1}(t, x), y_{2}(t, x)\right.$, $\left.\cdots, y_{n}(t, x)\right)^{T}$ with $y_{i}$ representing state variable related to a neuron. $J_{\sigma}=\left(J_{\sigma 1}, \cdots, J_{\sigma n}\right)^{T} \in \mathbb{R}^{n}$ is the constant external input vector, and both $D_{\sigma}$ and $C_{\sigma}$ are positive definite diagonal matrices, in which $D_{\sigma}$ represents the diffusion coefficient matrix, and $C_{\sigma}$ represents the connection weight matrix of neural network. Besides, $A_{\sigma}$ and $B_{\sigma}$ both are the connection weight matrices of neural network. For each $x \in \Omega_{\sigma}, g(y(t, x))=\left(g_{1}\left(y_{1}(t, x)\right), \cdots, g_{n}\left(y_{n}(t, x)\right)\right)^{T}$ represents a time-dependent signal function vector. $\tau(t)$ represents the time delay required for signal transmission from neuron $j$ to neuron $i$, satisfying $0 \leqslant \tau(t) \leqslant \tau$. Assumed that $y^{\sigma}(x)=\left(y_{1}^{\sigma}(x), \cdots, y_{n}^{\sigma}(x)\right)^{T}$ is a nontrivial stationary solution of reaction-diffusion switched system (2.1), then $y^{\sigma}(x)$ satisfies two equations of the system (2.1), in addition,

$$
-C_{\sigma} y^{\sigma}(x)+A_{\sigma} g\left(y^{\sigma}(x)\right)+B_{\sigma} g\left(y^{\sigma}(x)\right)+J_{\sigma} \not \equiv 0, \quad x \in \Omega_{\sigma} .
$$

Of course, the sufficient condition should be given to ensure the existence of such nontrivial stationary solution.

Set $u(t, x)=y(t, x)-y^{\sigma}(x)$, then the system (2.1) is translated into the following system:

$$
\left\{\begin{array}{l}
\frac{\partial u(t, x)}{\partial t}=D_{\sigma} \Delta u(t, x)-C_{\sigma} u(t, x)+A_{\sigma} f(u(t, x))+B_{\sigma} f(u(t-\tau(t), x)), \quad(t, x) \in \mathbb{R}_{+} \times \Omega_{\sigma}, \\
u_{i}(t, x)=0, t \geqslant 0, x \in \partial \Omega_{\sigma}, i=1,2, \cdots, n,
\end{array}\right.
$$

where $f(u(t, x))=g(y(t, x))-g\left(y^{\sigma}(x)\right), f(u(t-\tau(t), x))=g(y(t-\tau(t), x))-g\left(y^{\sigma}(x)\right)$. Here, the nontrivial stationary solution $y^{\sigma}(x)$ of the system (2.1) corresponds to the null solution of the system (2.3).

Besides, we may equip the system (2.3) with the initial value:

$$
u_{i}(s, x)=\phi_{i}(s, x),-\tau \leqslant s \leqslant 0, x \in \Omega_{\sigma},
$$

where $\left(\phi_{1}(s, x), \phi_{2}(s, x), \cdots, \phi_{n}(s, x)\right)^{T}=\phi(s, x)$, and each $\phi_{i}(s, x)$ is bounded on $[-\tau, 0] \times \Omega_{\sigma}$. 
In some cases, the following assumptions may be considered:

(A1) There is a positive definite diagonal matrix $G=\operatorname{diag}\left(G_{1}, G_{2}, \cdots, G_{n}\right)$ such that

$$
\left|g_{i}(s)-g_{i}(t)\right| \leqslant G_{i}|s-t|, \quad \forall s, t \in \mathbb{R}
$$

(A2) There is a positive real number $c>0$ such that

$$
0 \leqslant\left[-C_{\sigma} v+A_{\sigma} g(v)+B_{\sigma} g(v)+J_{\sigma}\right] \leqslant c D_{\sigma} E, \quad \forall v \in R^{n}
$$

where $D_{\sigma}>0$ is a positive definite diagonal matrix, and $E=(1,1, \cdots, 1)^{T} \in \mathbb{R}^{n}$.

Define the switching law as follows,

Switching Law $\mathfrak{F}$ : At each switching we determine the next mode according to the following minimum law :

$$
\sigma(t)=\arg \min \left(y-y^{\sigma}\right)^{T}\left[\left(-2 \lambda_{\sigma 1} D_{\sigma}-2 C_{\sigma}+A_{\sigma} A_{\sigma}^{T}+B_{\sigma} B_{\sigma}^{T}+G^{2}+e^{\gamma \tau} q G^{2}\right)+\Psi\right]\left(y-y^{\sigma}\right)
$$

$\left(\mathfrak{F}_{1}\right)$ Choose the initial mode $\sigma(t)=i_{0}$, if $\left(y\left(t_{0}, x\right)-y^{\sigma}(x)\right) \in \Upsilon_{i_{0}}$.

$\left(\mathfrak{F}_{2}\right)$ For each $t>t_{0}$, if $\sigma\left(t^{-}\right)=i$ and $\left(y-y^{\sigma}\right) \in \Upsilon_{i}$, keep $\sigma(t)=i$. On the other hand, if $\sigma\left(t^{-}\right)=i$ but $\left(y-y^{\sigma}\right) \notin \Upsilon_{i}$. i.e., hitting a switching surface, choose the next mode by applying (2.5) and begin to switch.

Here, $\Psi$ is a positive definite symmetric matrix with $\lambda_{\min } \Psi>0$, and $\Upsilon_{\sigma}$ is defined as follows,

$$
\Upsilon_{\sigma}=\left\{y \in \mathbb{R}^{n} \mid\left(y-y^{\sigma}\right)^{T}\left(-2 \lambda_{\sigma 1} D_{\sigma}-2 C_{\sigma}+A_{\sigma} A_{\sigma}^{T}+B_{\sigma} B_{\sigma}^{T}+G^{2}+e^{\gamma \tau} q G^{2}+\Psi\right)\left(y-y^{\sigma}\right)<0\right\}
$$

where $\lambda_{\min } \Psi$ represents the minimum of all the eigenvalues of the symmetric matrix $\Psi>0$.

Definition 1. A system is said to be globally asymptotically stable if it owns an equilibrium point which is globally asymptotically stable. Particularly, the globally asymptotical stability of an ordinary differential system is said to be invariant under the influence of diffusions if a constant equilibrium point $u^{*}$ of the ordinary differential system is globally asymptotically stable, and $u^{*}(x)$ is a globally asymptotically stable stationary solution of its corresponding reaction-diffusion system, where $u^{*}(x)$ is not necessarily equal to $u^{*}$.

Definition 2.([42]) Let $\psi$ be a real $C^{1}$ functional defined on a Banach space $X$. If any sequence $\left\{u_{n}\right\}$ in $X$ with $\psi\left(u_{n}\right) \rightarrow a$ and $\left\|\psi^{\prime}\left(u_{n}\right)\right\|_{X^{*}} \rightarrow 0$ has a convergent subsequence, and this holds for every $a \in \mathbb{R}$, one says that $\psi$ satisfies the (PS) condition.

Definition 3. Suppose that for each $\sigma \in \mathfrak{T}$, there exists the unique stationary solution $y^{\sigma}(x)$ for the switched system (2.1), and $u=y(t, x)-y^{\sigma}(x)$ satisfies

$$
\|u\|_{L^{2}\left(\Omega_{\sigma}\right)}^{2} \leqslant M\|\phi\|_{\tau}^{2} e^{-\gamma t}, \quad \forall t \geqslant 0
$$

where $\gamma>0$ and $M>1$ are constants. Then we say, the switched system (2.1) is globally exponentially stable in the meaning of switching, and the null solution the null solution of the switched delayed reaction-diffusion system (2.3) 
equipped with the initial value (2.4) is globally exponentially stable. Particularly, in the case of $\mathfrak{T}=\{1\}$ or $y^{\sigma}(x) \equiv y(x)$ for all $\sigma \in \mathfrak{T}$, we say, the switched system (2.1) is globally exponentially stable (in the classical meaning).

Lemma 2.1.([32]) Let $\mathfrak{Z}$ be a Banach space, and $\mathfrak{K}$ is a closed convex set. If $\mathfrak{T}: \mathfrak{K} \rightarrow \mathfrak{K}$ is a compact mapping such that for any $\varphi \in \mathfrak{K}$ with $\|\varphi\|=M$, the inequality $\varphi \neq r \mathfrak{T}(\varphi)$ holds for each $r \in[0,1]$, where $M$ is any given positive constant, then there exits at least a fixed point of $\mathfrak{T}$, say, $\varphi \in \mathfrak{K}$ with $\|\varphi\| \leqslant M$.

Lemma 2.2. ([42]) Let $H=H_{1} \oplus H_{2}$ be a Banach space, and $H_{1}$ is a finite dimension subspace. If $\psi \in C^{1}(H, \mathbb{R})$, satisfying $\psi(0)=0$, the (PS) condition. Besides, for some $\delta>0$, the following conditions hold,

(P1) $\psi(u) \leqslant 0$ if $u \in H_{1}$ with $\|u\| \leqslant \delta$;

(P2) $\psi(u) \geqslant 0$ if $u \in H_{2}$ with $\|u\| \leqslant \delta$;

(P3) $\psi$ is bounded below, satisfying $\inf _{H} \psi<0$,

then $\psi$ owns at least two non-zero critical points.

Lemma 2.3 ([48]). For the given matrices $E, F$, and $G$ with $F^{T} F \leqslant I$ and scalar $\varepsilon>0$, the following inequality holds:

$$
G F E+E^{T} F^{T} G^{T} \leqslant \varepsilon G G^{T}+\varepsilon E^{T} E
$$

\section{Main results}

Theorem 3.1. Suppose that the conditions (A1) and (A2) hold, then the system (2.1) possesses a positive bounded stationary solution $y^{\sigma}(x)$ for $x \in \Omega_{\sigma}$ with $\left.y^{\sigma}\right|_{\partial \Omega_{\sigma}}=0$. In addition, there is a sequence of nonnegative constants $\beta_{\sigma}(\sigma=1,2, \cdots, N)$ with $\sum_{\sigma=1}^{N} \beta_{\sigma}=1$ and $0 \leqslant \beta_{\sigma} \leqslant 1$ and positive constants $\gamma \in\left(0, \lambda_{\min } \Psi\right)$ and $q>1$ such that

$$
\sum_{\sigma=1}^{N} \beta_{\sigma}\left(-2 \lambda_{\sigma 1} D_{\sigma}-2 C_{\sigma}+A_{\sigma} A_{\sigma}^{T}+B_{\sigma} B_{\sigma}^{T}\right)+G^{2}+e^{\gamma \tau} q G^{2}+\Psi<0,
$$

then the null solution of the switched delayed reaction-diffusion system (2.3) equipped with the initial value (2.4) is exponentially stable with the convergence rate $\frac{\gamma}{2}$.

Proof. Firstly, we denote $\left\|u_{i}\right\|=\sqrt{\int_{\Omega_{\sigma}}\left|\nabla u_{i}\right|^{2} d x}$, and $\|u\|=\sum_{i=1}^{n}\left\|u_{i}\right\|$. Besides, denote by $I$ the identity matrix. If the stationary solution of the system (2.1) exists, we may denote $y^{\sigma}(x)$.

Define the operator $\mathfrak{M}:\left[C\left(\overline{\Omega_{\sigma}}\right)\right]^{n} \rightarrow\left[C\left(\overline{\Omega_{\sigma}}\right)\right]^{n}$ as follows,

$$
\mathfrak{M}=\left(\begin{array}{ccccc}
-\Delta & 0 & 0 & \cdots & 0 \\
0 & -\Delta & 0 & \cdots & 0 \\
\vdots & \vdots & \vdots & \cdots & \vdots \\
0 & 0 & 0 & \cdots & -\Delta
\end{array}\right) .
$$


The operator $\mathfrak{M}$ has the inverse operator $\mathfrak{M}^{-1}$ as follows,

$$
\mathfrak{M}^{-1}=\left(\begin{array}{ccccc}
(-\Delta)^{-1} & 0 & 0 & \cdots & 0 \\
0 & (-\Delta)^{-1} & 0 & \cdots & 0 \\
\vdots & \vdots & \vdots & \cdots & \vdots \\
0 & 0 & 0 & \cdots & (-\Delta)^{-1}
\end{array}\right)
$$

where $\mathfrak{M}^{-1}:\left[C\left(\overline{\Omega_{\sigma}}\right)\right]^{n} \rightarrow\left[C\left(\overline{\Omega_{\sigma}}\right)\right]^{n}$ is a linear compact positive operator $([13])$, and

$$
\left\{\begin{array}{l}
\mathfrak{M} y^{\sigma}(x)=-D_{\sigma}^{-1} C_{\sigma} y^{\sigma}(x)+D_{\sigma}^{-1} A_{\sigma} g\left(y^{\sigma}(x)\right)+D_{\sigma}^{-1} B_{\sigma} g\left(y^{\sigma}(x)\right)+D_{\sigma}^{-1} J_{\sigma}, \quad x \in \Omega_{\sigma} \\
y_{i}^{\sigma}(x)=0, x \in \partial \Omega_{\sigma}, i=1,2, \cdots, n
\end{array}\right.
$$

It is obvious that $\left(-D_{\sigma}^{-1} C_{\sigma} y^{\sigma}(x)+D_{\sigma}^{-1} A_{\sigma} g\left(y^{\sigma}(x)\right)+D_{\sigma}^{-1} B_{\sigma} g\left(y^{\sigma}(x)\right)+D_{\sigma}^{-1} J_{\sigma}\right)$ is continuous for all the variables $x, y_{1}^{\sigma}, \cdots, y_{n}^{\sigma}$. Define

$$
\mathfrak{K}=\left\{\varphi(x) \in\left[C\left(\overline{\Omega_{\sigma}}\right)\right]^{n}: \varphi(x) \geqslant 0, x \in \Omega ; \varphi(x)=0, x \in \partial \Omega\right\},
$$

then $\mathfrak{K}$ is a positive cone, which must be a closed convex subset of $\left[C\left(\overline{\Omega_{\sigma}}\right)\right]^{n}$. Define an operator $\mathfrak{T}: \mathfrak{K} \rightarrow \mathfrak{K}$ such that

$$
\mathfrak{T} \varphi=\mathfrak{M}^{-1}\left(-D_{\sigma}^{-1} C_{\sigma} \varphi+D_{\sigma}^{-1} A_{\sigma} g(\varphi)+D_{\sigma}^{-1} B_{\sigma} g(\varphi)+D_{\sigma}^{-1} J_{\sigma}\right), \quad \varphi \in \mathfrak{K}
$$

Because $\mathfrak{M}^{-1}$ is the linear positive compact operator([13]), and $\left(-D_{\sigma}^{-1} C_{\sigma} y^{\sigma}(x)+D_{\sigma}^{-1} A_{\sigma} g\left(y^{\sigma}(x)\right)+D_{\sigma}^{-1} B_{\sigma} g\left(y^{\sigma}(x)\right)+\right.$ $\left.D_{\sigma}^{-1} J_{\sigma}\right)$ is positive continuous, we can conclude that $\mathfrak{T}: \mathfrak{K} \rightarrow \mathfrak{K}$ is a positive compact operator.

Next, we claim that $\mathfrak{T}$ satisfies all the assumption conditions of Lemma 2.1, which implies that $\mathfrak{T}$ has at least a fixed point in $\mathfrak{K}$.

Indeed, if it is not true, there must be $\left\{r_{n}\right\} \subset[0,1]$ and $\left\{\varphi_{n}\right\} \subset \mathfrak{K}$ with

$$
\varphi_{n}=r_{n} \mathfrak{T}\left(\varphi_{n}\right)=r_{n} \mathfrak{M}^{-1}\left(-D_{\sigma}^{-1} C_{\sigma} \varphi_{n}+D_{\sigma}^{-1} A_{\sigma} g\left(\varphi_{n}\right)+D_{\sigma}^{-1} B_{\sigma} g\left(\varphi_{n}\right)+D_{\sigma}^{-1} J_{\sigma}\right)
$$

and

$$
\left\|\varphi_{n}\right\|=M_{n} \rightarrow+\infty, \quad n \rightarrow+\infty
$$

The compactness of bounded closed sets in a finite dimensional space yields that there is a subsequence of $\left\{r_{n}\right\}$, say, $\left\{r_{n}\right\}$ such that $\lim _{n \rightarrow \infty} r_{n}=r_{0}$.

Let

$$
\mathfrak{L}_{n}=\frac{\varphi_{n}}{\left\|\varphi_{n}\right\|}
$$

then it is easy to conclude from (3.4) and (H2) that if $r_{n} \rightarrow r_{0} \in[0,1]$,

$$
\mathfrak{L}_{n} \rightarrow \mathfrak{L}_{0} \in \mathfrak{K}, \quad\left\|\mathfrak{L}_{0}\right\|=1 .
$$


In fact, combining $(\mathrm{H} 2)$ and the property of the operator $\mathfrak{M}^{-1}$ yields

$$
\mathfrak{L}_{n}=r_{n} \mathfrak{M}^{-1}\left(\frac{-D_{\sigma}^{-1} C_{\sigma} \varphi_{n}+D_{\sigma}^{-1} A_{\sigma} g\left(\varphi_{n}\right)+D_{\sigma}^{-1} B_{\sigma} g\left(\varphi_{n}\right)+D_{\sigma}^{-1} J_{\sigma}}{\left\|\varphi_{n}\right\|}\right) \rightarrow 0 \in R^{n}, \quad n \rightarrow \infty .
$$

On one hand, $\mathfrak{L}_{0}=0$ implies $\left\|\mathfrak{L}_{0}\right\|=0$. On the other hand, it follows by $\mathfrak{L}_{n} \rightarrow \mathfrak{L}_{0}$ and $\left\|\mathfrak{L}_{n}\right\|=1$ that $\left\|\mathfrak{L}_{0}\right\|=1$, which contradicts $\left\|\mathfrak{L}_{0}\right\|=0$, and hence all the conditions of Lemma 2.1 are satisfied. Thereby, there exists $y^{\sigma} \in \mathfrak{K}$ such that $y^{\sigma}=\mathfrak{T} y^{\sigma}$ with $\left\|y^{\sigma}\right\| \leqslant M$, and $y^{\sigma}$ is a bounded positive solution of the system (2.1).

Next, we consider the following Lyapunov functional :

$$
V=\int_{\Omega_{\sigma}}\left[y(t, x)-y^{\sigma}(x)\right]^{T}\left[y(t, x)-y^{\sigma}(x)\right] d x=\int_{\Omega_{\sigma}} u^{T}(t, x) u(t, x) d x .
$$

Set

$$
U(t, u(t, x))= \begin{cases}e^{\gamma t} \int_{\Omega_{\sigma}} u^{T}(t, x) u(t, x) d x, & t \geqslant 0 \\ \int_{\Omega_{\sigma}} u^{T}(t, x) u(t, x) d x, & t \in[-\tau, 0],\end{cases}
$$

It is obvious that $U$ is continuous for $t \geqslant-\tau$. For $t \geqslant 0$ and $\gamma>0$,

Now we claim that there is a positive constants $C_{0}>1$ and $K \in \mathbb{R}$ with $K>1$ such that

$$
U(t, u(t, x)) \leqslant K C_{0}\|\phi\|_{\tau}^{2}, \quad \forall t \geqslant 0,
$$

where $\|\phi\|_{\tau}^{2}=\sup _{s \in[-\tau, 0]} \int_{\Omega_{\sigma}} \phi^{T}(s, x) \phi(s, x) d x$.

Indeed, suppose this claim is not true, then there must be a $t \geqslant 0$ such that $U(t, u(t, x))>K C_{0}\|\phi\|_{\tau}^{2}$. Obviously, (3.9) holds for $t \in[-\tau, 0]$, and hence there must exist $t^{*}>0$ such that

$$
U\left(t^{*}, u\left(t^{*}, x\right)\right)=K C_{0}\|\phi\|_{\tau}^{2} \quad \text { and } \quad U(t, u(t, x)) \leqslant K C_{0}\|\phi\|_{\tau}^{2}, \forall t \in\left[0, t^{*}\right],
$$

and hence

$$
U\left(t^{*}, u\left(t^{*}, x\right)\right)=K C_{0}\|\phi\|_{\tau}^{2} \quad \text { and } \quad U(t, u(t, x)) \leqslant K C_{0}\|\phi\|_{\tau}^{2}, \forall t \in\left[-\tau, t^{*}\right] .
$$

Let $q>1$, and due to $U(0, u(0, x))<K C_{0}\|\phi\|_{\tau}^{2}=U\left(t^{*}, u\left(t^{*}, x\right)\right)$, there is $t^{* *} \in\left[0, t^{*}\right]$ such that

$$
\left\{\begin{array}{l}
U\left(t^{* *}, u\left(t^{* *}\right)\right)=\frac{1}{q} K C_{0}\|\phi\|_{\tau}^{2}<K C_{0}\|\phi\|_{\tau}^{2}=U\left(t^{*}, u\left(t^{*}\right)\right) ; \\
U\left(t^{* *}, u\left(t^{* *}\right)\right) \leqslant U(t, u(t, x)) \leqslant U\left(t^{*}, u\left(t^{*}\right)\right)=K C_{0}\|\phi\|_{\tau}^{2}, \quad \forall t \in\left[t^{* *}, t^{*}\right] .
\end{array}\right.
$$

It follows from (3.10), (3.11) and the definition of $U(t, u(t, x))$ that for $s \in[-\tau, 0]$ and $t \in\left[t^{* *}, t^{*}\right]$,

$$
\begin{aligned}
& \int_{\Omega_{\sigma}} e^{\gamma s}\left[u^{T}(t+s) u(t+s)\right] d x \\
= & \left\{\begin{array}{cc}
e^{-\gamma t} U(t+s, u(t+s)), & t+s \geqslant 0 \\
e^{\gamma s} U(t+s, u(t+s)), & t+s \leqslant 0
\end{array}\right. \\
\leqslant & e^{-\gamma t} U(t+s, u(t+s)) \\
\leqslant & q \int_{\Omega_{\sigma}}\left[u^{T}(t, x) u(t, x)\right] d x,
\end{aligned}
$$


which yields that for any $s \in[-\tau, 0]$,

$$
\int_{\Omega_{\sigma}}\left[u^{T}(t-\tau(t)) u(t-\tau(t))\right] d x \leqslant e^{\gamma \tau} q \int_{\Omega_{\sigma}} u^{T}(t, x) u(t, x) d x, \quad t \in\left[t^{* *}, t^{*}\right] .
$$

On the other hand, the condition (A1) yields

$$
\begin{aligned}
u^{T} A_{\sigma} f(u)+f^{T}(u) A_{\sigma}^{T} u & =\left(A_{\sigma}^{T} u\right)^{T} f(u)+f^{T}(u)\left(A_{\sigma}^{T} u\right) \\
& \leqslant u^{T}\left(A_{\sigma} A_{\sigma}^{T}\right) u+u^{T} G^{2} u
\end{aligned}
$$

and

$$
u^{T} B_{\sigma} f(u(t-\tau(x), x))+f^{T}(u(t-\tau(x), x)) B_{\sigma}^{T} u \leqslant u^{T}\left(B_{\sigma} B_{\sigma}^{T}\right) u+u^{T}(t-\tau(x), x) G^{2} u(t-\tau(x), x)
$$

Now, we calculate the derivative $\frac{d V}{d t}$ alongside with the trajectories of the system (2.1) or (2.3) as follows,

$$
\begin{aligned}
\frac{d V}{d t}= & 2 \int_{\Omega_{\sigma}} u^{T}(t, x)\left[D_{\sigma} \Delta u(t, x)-C_{\sigma} u(t, x)+A_{\sigma} f(u(t, x))+B_{\sigma} f(u(t-\tau(t), x))\right] d x \\
\leqslant & \int_{\Omega_{\sigma}} u^{T}(t, x)\left(-2 \lambda_{1} D_{\sigma}-2 C_{\sigma}+A_{\sigma} A_{\sigma}^{T}+B_{\sigma} B_{\sigma}^{T}+G^{2}\right) u(t, x) d x \\
& +\int_{\Omega_{\sigma}} u^{T}(t-\tau(x), x) G^{2} u(t-\tau(x), x) d x,
\end{aligned}
$$

which together with (3.13) implies that

$$
\frac{d V}{d t} \leqslant \int_{\Omega_{\sigma}} u^{T}(t, x)\left(-2 \lambda_{1} D_{\sigma}-2 C_{\sigma}+A_{\sigma} A_{\sigma}^{T}+B_{\sigma} B_{\sigma}^{T}+G^{2}+e^{\gamma \tau} q G^{2}\right) u(t, x) d x, t \in\left[t^{* *}, t^{*}\right] .
$$

For any given $t \geqslant t_{0}$, according to the switching law $\mathfrak{F}$, when $\sigma\left(t^{-}\right)=i$ and $u(t, x) \in \Upsilon_{i}$, then keep $\sigma(t)=i$, and we can conclude that

$$
\begin{aligned}
\frac{d V}{d t} & \leqslant \int_{\Omega_{\sigma}} u^{T}(t, x)\left(-2 \lambda_{1} D_{\sigma}-2 C_{\sigma}+A_{\sigma} A_{\sigma}^{T}+B_{\sigma} B_{\sigma}^{T}+G^{2}+e^{\gamma \tau} q G^{2}\right) u(t, x) d x \\
& \leqslant-\lambda_{\min } \Psi V(t, u(t, x)), t \in\left[t^{* *}, t^{*}\right] .
\end{aligned}
$$

When $\sigma\left(t^{-}\right)=i$ and $u(t, x) \notin \Upsilon_{i}$, which means that the trajectory hits a switching surface. On the other hand, it is not difficult to deduce from (3.1) that $\bigcup_{i=1}^{N} \Upsilon_{i}=R^{n} \backslash\{0\}$, which together with the minimum law (2.5) yields (3.15), too.

Thereby, it follows from the definition of $U(t, u(t, x))$ that

$$
\frac{d U}{d t}=\left(\gamma-\lambda_{\min } \Psi\right) U\left(t, u(t, x) \leqslant 0, t \in\left[t^{* *}, t^{*}\right]\right.
$$

which derives that $U\left(t^{*}, u\left(t^{*}\right)\right) \leqslant U\left(t^{* *}, u\left(t^{* *}\right)\right)$. This contradicts (3.11). So we have prove the claim (3.9), which means

$$
e^{\gamma t} \int_{\Omega_{\sigma}} u^{T}(t, x) u(t, x) d x \leqslant K C_{0}\|\phi\|_{\tau}^{2}, \quad \forall t \geqslant 0
$$

or

$$
\|u\|_{L^{2}\left(\Omega_{\sigma}\right)}^{2} \leqslant K C_{0}\|\phi\|_{\tau}^{2} e^{-\gamma t}, \quad \forall t \geqslant 0
$$

which implies that the switched delayed reaction-diffusion system (2.3) equipped with the initial value (2.4) is exponentially stable with the convergence rate $\frac{\gamma}{2}$. 
Remark 1. Particularly, in the case of $\mathfrak{T}=\{1\}$ or $N=1$, the system (2.1) is the common delayed reaction-diffusion system without any switches. Theorem 3.1 includes the exponential stability in the classical sense for the positive bounded stationary solution of the following common delayed reaction-diffusion system:

$$
\left\{\begin{array}{l}
\frac{\partial y(t, x)}{\partial t}=D \Delta y(t, x)-C y(t, x)+A g(y(t, x))+B g(y(t-\tau(t), x))+J, \quad(t, x) \in \mathbb{R}_{+} \times \Omega, \\
y_{i}(t, x)=0, t \geqslant 0, x \in \partial \Omega, i=1,2, \cdots, n .
\end{array}\right.
$$

Remark 2. For the first time, Theorem 3.1 shows the two sides of the diffusion phenomena in practical engineering (see Remark 5 for details). But [1, Theorem 1] only shows one side of the diffusion phenomena which promotes the stability of reaction-diffusion neural networks. So do those of previous related literature [2-10] and the references therein.

Next, the uniqueness of the positive stationary solution of Theorem 3.1 will be presented by adding a condition to Theorem 3.1 so that the exponential stability of the positive stationary solution is global (in the meaning of Definition 3).

Theorem 3.2. If all the assumptions of Theorem 3.1 hold, and if, in addition, the following condition is satisfied,

(A3) for each $\sigma \in \mathfrak{T}$, there exists a scalar $\varepsilon>0$ such that

$$
-C_{\sigma}+\frac{p_{\sigma}}{2}\left(\varepsilon^{-1} I+\varepsilon G^{2}\right)<\lambda_{\sigma 1} D_{\sigma}
$$

where the constant $p_{\sigma}>0$ satisfying $p_{\sigma}^{2} I \geqslant\left(A_{\sigma}+B_{\sigma}\right)^{T}\left(A_{\sigma}+B_{\sigma}\right)$, then the system (2.1) possesses a unique positive bounded stationary solution $y^{\sigma}(x)$ for $x \in \Omega_{\sigma}$ with $\left.y^{\sigma}\right|_{\partial \Omega_{\sigma}}=0$. And the null solution of the switched delayed reactiondiffusion system (2.3) equipped with the initial value (2.4) is globally exponentially stable with the convergence rate $\frac{\gamma}{2}$. Particulary, if $\mathfrak{T}=\{1\}$ or $N=1$, the unique stationary solution $y^{\sigma}(x)(\sigma=1)$ of the deterministic system $(2.1)$ is globally exponentially stable in the classical meaning.

Proof. Assume both $y(x)$ and $v(x)$ are the stationary solutions of the system (2.1). Then we claim $y(x)=v(x)$.

In fact, Lemma 2.3 yields

$$
\begin{aligned}
& (y(x)-v(x))^{T}\left(A_{\sigma}+B_{\sigma}\right)(g(y(x))-g(v(x))) \\
= & \frac{1}{2}\left[(y(x)-v(x))^{T}\left(A_{\sigma}+B_{\sigma}\right)(g(y(x))-g(v(x)))+(g(y(x))-g(v(x)))^{T}\left(A_{\sigma}+B_{\sigma}\right)^{T}(y(x)-v(x))\right] \\
\leqslant & \frac{p_{\sigma}}{2}(y(x)-v(x))^{T}\left[\varepsilon^{-1} I+\varepsilon G^{2}\right](y(x)-v(x)) .
\end{aligned}
$$

Since both $y(x)$ and $v(x)$ are the stationary solutions of the system (2.1), we can see it from (3.18), variational method 
and the Poincare inequality that

$$
\begin{aligned}
& \lambda_{\sigma 1} \int_{\Omega_{\sigma}}|y(x)-v(x)|^{T} D_{\sigma}|y(x)-v(x)| d x \leqslant \int_{\Omega_{\sigma}}|\nabla(y(x)-v(x))|^{T} D_{\sigma}|\nabla(y(x)-v(x))| d x \\
\leqslant & -\int_{\Omega_{\sigma}}|y(x)-v(x)|^{T} C_{\sigma}|y(x)-v(x)| d x+\int_{\Omega_{\sigma}} \frac{p_{\sigma}}{2}(y(x)-v(x))^{T}\left[\varepsilon^{-1} I+\varepsilon G^{2}\right](y(x)-v(x)) d x \\
\leqslant & \int_{\Omega_{\sigma}}|y(x)-v(x)|^{T}\left[-C_{\sigma}+\frac{p_{\sigma}}{2}\left(\varepsilon^{-1} I+\varepsilon G^{2}\right)\right]|y(x)-v(x)| d x .
\end{aligned}
$$

Now the condition (A3) yields the claim via the proof by contradiction. And so the system (2.1) possesses a unique positive bounded stationary solution $y^{\sigma}(x)$ for $x \in \Omega_{\sigma}$ with $\left.y^{\sigma}\right|_{\partial \Omega_{\sigma}}=0$. Moreover, according to the proof of Theorem 3.1, the unique positive bounded stationary solution $y^{\sigma}(x)$ is globally exponentially stable, i.e., the null solution of the switched delayed reaction-diffusion system (2.3) equipped with the initial value (2.4) is globally exponentially stable with the convergence rate $\frac{\gamma}{2}$.

Remark 3. (A2) and (A3) are the sufficient conditions, guaranteeing the global stability invariance of ordinary differential systems under the influence of diffusions in the meaning of Definition 1 (see Corollary 3.4).

To show the idea of Remark 2, we may consider the stability of the constant equilibrium point of the following delayed reaction-diffusion Cohen-Grossberg neural networks which is the partial differential equations model studied in [2]:

$$
\left\{\begin{array}{l}
\frac{\partial u_{i}(t, x)}{\partial t}=r_{i} \Delta u_{i}(t, x)-a_{i}\left(u_{i}(t, x)\right)\left[b_{i}\left(u_{i}(t, x)\right)-\sum_{j=1}^{n} c_{i j} f_{j}\left(u_{j}(t, x)\right)-\sum_{j=1}^{n} d_{i j} g_{j}\left(u_{j}\left(t-\tau_{j}(t), x\right)\right)+I_{i}\right], \quad t \geqslant 0, t \neq t_{k}, x \in \Omega, \\
u_{i}\left(t^{+}, x\right)=m_{i} u_{i}\left(t^{-}, x\right)+\sum_{j=1}^{n} n_{i j} h_{j}\left(u_{j}\left(t^{-}-\tau_{j}(t), x\right)\right), \quad t=t_{k}, 0 \leqslant \tau_{j}(t) \leqslant \tau_{j}, \forall j, \\
u_{i}(t, x)=0, \quad t \geqslant 0, x \in \partial \Omega, i=1,2, \cdots, n, \\
u_{i}(s, x)=\phi_{i}(s, x), \quad-\tau \leqslant s \leqslant 0, \tau=\max _{1 \leqslant j \leqslant n} \tau_{j},
\end{array}\right.
$$

where $u_{i}\left(t_{k}^{+}, x\right)=u_{i}\left(t_{k}, x\right)$, all the variables, coefficients and functions are defined in [2], and are different from those of our Theorem 3.1 and Theorem 3.2. Below, we will give a stability criterion of its corresponding ordinary differential equations as follows, which will be completely similar as [2, Theorem 3.1]:

$$
\left\{\begin{array}{l}
\frac{d u_{i}(t)}{d t}=-a_{i}\left(u_{i}(t)\right)\left[b_{i}\left(u_{i}(t)\right)-\sum_{j=1}^{n} c_{i j} f_{j}\left(u_{j}(t)\right)-\sum_{j=1}^{n} d_{i j} g_{j}\left(u_{j}\left(t-\tau_{j}(t)\right)\right)+I_{i}\right], \quad t \geqslant 0, t \neq t_{k} \\
u_{i}\left(t^{+}\right)=m_{i} u_{i}\left(t^{-}\right)+\sum_{j=1}^{n} n_{i j} h_{j}\left(u_{j}\left(t^{-}-\tau_{j}(t)\right)\right), \quad t=t_{k} \\
u_{i}(s)=\phi_{i}(s), \quad-\tau \leqslant s \leqslant 0, \quad \tau=\max _{1 \leqslant j \leqslant n} \tau_{j}, \quad i=1,2, \cdots, n
\end{array}\right.
$$

For the convenience of readers, we may copy the assumption conditions in the document [2] as follows,

(H1) Each function $a_{i}(u)$ is bounded, positive and continuous, i.e., there exist two positive diagonal matrices 
$\underline{A}=\operatorname{diag}\left(\underline{A}_{1}, \underline{A}_{2}, \cdots, \underline{A}_{n}\right)$ and $\underline{A}=\operatorname{diag}\left(\bar{A}_{1}, \bar{A}_{2}, \cdots, \bar{A}_{n}\right)$ such that

$$
0<\underline{A}_{i} \leqslant a_{i}(u) \leqslant \bar{A}_{i}, \quad \forall u \in \mathbb{R}, \forall i .
$$

(H2) Each function $b_{i}(u)$ is monotone increasing, i.e., there exist a positive diagonal matrix $B=\operatorname{diag}\left(B_{1}, B_{2}, \cdots, B_{n}\right)$ such that

$$
\frac{b_{i}(u)-b_{i}(v)}{u-v} \geqslant B_{i}, \quad \forall u, v(u \neq v) \in \mathbb{R}, \forall i .
$$

(H3) There exist three positive diagonal matrices $F=\operatorname{diag}\left(F_{1}, F_{2}, \cdots, F_{n}\right), G=\operatorname{diag}\left(G_{1}, G_{2}, \cdots, G_{n}\right)$ and $H=$ $\operatorname{diag}\left(H_{1}, H_{2}, \cdots, H_{n}\right)$ such that

$$
0 \leqslant \frac{f_{i}(u)-f_{i}(v)}{u-v} \leqslant F_{i}, \quad 0 \leqslant \frac{g_{i}(u)-g_{i}(v)}{u-v} \leqslant G_{i}, \quad 0 \leqslant \frac{h_{i}(u)-h_{i}(v)}{u-v} \leqslant H_{i}, \quad \forall u, v(u \neq v) \in \mathbb{R}, \forall i .
$$

It is obvious that the system (3.19) and its corresponding ordinary differential equations (3.20) own the same constant equilibrium point $u^{*}=\left(u_{1}^{*}, \cdots, u_{n}^{*}\right)^{T} \in \mathbb{R}^{n}$ if $u^{*}=\left(u_{1}^{*}, \cdots, u_{n}^{*}\right)^{T}$ satisfies

$$
\left\{\begin{array}{l}
b_{i}\left(u_{i}^{*}\right)-\sum_{j=1}^{n} c_{i j} f_{j}\left(u_{j}^{*}\right) \sum_{j=1}^{n} d_{i j} g_{j}\left(u_{j}^{*}\right)+I_{i}=0, \\
\left(m_{i}-1\right) u_{i}^{*}+\sum_{j=1}^{n} n_{i j} h_{j}\left(u_{j}^{*}\right)=0,
\end{array} \quad \forall i, j=1,2, \cdots, n .\right.
$$

Theorem 3.3. Under assumptions (H1)-(H3), if the following conditions hold:

$(\tilde{C} 1)$ there exists a positive diagonal matrix $P>0$ such that

$$
\widetilde{\Psi}=\left(\begin{array}{ccc}
-2 P \underline{A} B+F^{2} & P \bar{A}|C| & P \bar{A}|D| \\
\left|C^{T}\right| \bar{A} P & -I & 0 \\
\left|D^{T}\right| \bar{A} P & 0 & -I
\end{array}\right)<0,
$$

where $R=\operatorname{diag}\left(r_{1}, r_{2}, \cdots, r_{n}\right),|C|=\left(\left|c_{i j}\right|\right)_{n \times n},|D|=\left(\left|d_{i j}\right|\right)_{n \times n}, I=\operatorname{diag}(1,1, \cdots, 1)$;

(C) $\tilde{a}=\frac{\lambda_{\min } \widetilde{\Phi}}{\lambda_{\max } P}>\frac{\lambda_{\max } G^{2}}{\lambda_{\min } P}=b \geqslant 0$, where

$$
\widetilde{\Phi}=2 P \underline{A} B-P \bar{A}|C|\left|C^{T}\right| \bar{A} P-P \bar{A}\left|D \| D^{T}\right| \bar{A} P-F^{2}>0 ;
$$

(C3) there exists a constant $\delta$ such that $\delta>\ln \left(\rho e^{\lambda \tau}\right) / \delta \tau$, where $\lambda>0$ is the unique solution of the equation $\lambda=a-$ $b e^{\lambda \tau}$, and $\rho=\max \left\{1, \frac{2 \lambda_{\max }(P M P)}{\lambda_{\min } P}+\frac{2 \lambda_{\max }\left(H N^{T} P N H\right)}{\lambda_{\min } P} e^{\lambda \tau}\right\}, M=\operatorname{diag}\left(m_{1}, m_{2}, \cdots, m_{n}\right), N=\left(n_{i j}\right)_{n \times n}, H=\operatorname{diag}\left(H_{1}, \cdots, H_{n}\right)$;

then we have the conclusions:

Conclusion (1) the constant equilibrium point $u^{*}$ of the ordinary differential system (3.20) is globally exponentially stable with convergence rate $\frac{1}{2}\left(\lambda-\frac{\ln \left(\rho e^{\ell \tau}\right)}{\delta \tau}\right)$; 
Conclusion (2) If there exits a solution $u^{*}(x)\left(\neq u^{*}\right.$ in common cases $)$ of the following equations:

$$
\left\{\begin{aligned}
& 0=r_{i} \Delta u_{i}(x)-a_{i}\left(u_{i}(x)\right)\left[b_{i}\left(u_{i}(x)\right)-\sum_{j=1}^{n} c_{i j} f_{j}\left(u_{j}(x)\right)-\sum_{j=1}^{n} d_{i j} g_{j}\left(u_{j}(x)\right)+I_{i}\right], \quad x \in \Omega \\
& u_{i}(x)=m_{i} u_{i}(x)+\sum_{j=1}^{n} n_{i j} h_{j}\left(u_{j}(x)\right), \quad \forall j, \\
& u_{i}(x)=0, \quad x \in \partial \Omega, i=1,2, \cdots, n,
\end{aligned}\right.
$$

then the globally exponential stability of the equilibrium point $u^{*}$ of the reaction-diffusion system (3.20) directly yields that the stationary solution $u^{*}(x)$ of the system (3.19) is also globally exponentially stable with the same convergence rate $\frac{1}{2}\left(\lambda-\frac{\ln \left(\rho e^{\lambda \tau}\right)}{\delta \tau}\right)$, which implies that the diffusion promotes the stability, or the diffusion is not harmful to the stability.

Proof. Firstly, we may prove the first conclusion of Theorem 3.1 involved in the system (3.20).

Next, for any given $i$, let $y_{i}(t)=u_{i}(t)-u_{i}^{*}$, where $u^{*}$ is the constant equilibrium point of the system (3.20). Then the system (3.20) can be transformed into

$$
\left\{\begin{array}{l}
\frac{d y_{i}(t)}{d t}=-\tilde{a}_{i}\left(y_{i}(t)\right)\left[\tilde{b}_{i}\left(y_{i}(t)\right)-\sum_{j=1}^{n} c_{i j} \tilde{f}_{j}\left(y_{j}(t)\right)-\sum_{j=1}^{n} d_{i j} \tilde{g}_{j}\left(y_{j}\left(t-\tau_{j}(t)\right)\right)\right], \quad t \geqslant 0, t \neq t_{k}, \\
y_{i}\left(t^{+}\right)=m_{i} y_{i}\left(t^{-}\right)+\sum_{j=1}^{n} n_{i j} \tilde{h}_{j}\left(y_{j}\left(t^{-}-\tau_{j}(t)\right)\right), \quad t=t_{k}, \\
y_{i}(s)=\phi_{i}(s)-u_{i}^{*}(s), \quad-\tau \leqslant s \leqslant 0, \quad \tau=\max _{1 \leqslant j \leqslant n} \tau_{j}, \quad i=1,2, \cdots, n .
\end{array}\right.
$$

where $\tilde{a}_{i}\left(y_{i}(t)\right)=a_{i}\left(y_{i}(t)+u_{i}^{*}\right), \tilde{b}_{i}\left(y_{i}(t)\right)=b_{i}\left(y_{i}(t)+u_{i}^{*}\right)-b_{i}\left(u_{i}^{*}\right), \tilde{f}_{j}\left(y_{j}(t)\right)=f_{j}\left(y_{j}(t)+u_{j}^{*}\right)-f_{j}\left(u_{j}^{*}\right), \tilde{g}_{j}\left(y_{j}(t)\right)=$ $g_{j}\left(y_{j}(t)+u_{j}^{*}\right)-g_{j}\left(u_{j}^{*}\right), \tilde{h}_{j}\left(y_{j}(t)\right)=h_{j}\left(y_{j}(t)+u_{j}^{*}\right)-h_{j}\left(u_{j}^{*}\right)$ for all $i, j=1,2, \cdots, n$.

Similarly as the proof of [2, Theorem 3.1], we may set up the Lyapunov function as follows,

$$
\widetilde{V}(t)=Y^{T}(t) P Y(t)=\left|Y^{T}(t)\right| P|Y(t)|
$$

where $Y(t)=\left(y_{1}(t), \cdots, y_{n}(t)\right)^{T}, P=\operatorname{diag}\left(p_{1}, p_{2}, \cdots, p_{n}\right)$.

For the case of $t \neq t_{k}$, we compute the Dini derivative of $\widetilde{V}(t)$ alongside with the trajectories of (3.23),

$$
\begin{aligned}
D^{+} \widetilde{V}(t) & =-2 Y^{T}(t) P \widetilde{A}(Y(t)) \widetilde{B}(Y(t))+2 Y^{T}(t) P \widetilde{A}(Y(t)) C \widetilde{F}(Y(t))+2 Y^{T}(t) P \widetilde{A}(Y(t)) D \widetilde{G}(Y(t-\tau(t))) \\
& \leqslant-\left|Y^{T}(t)\right| \widetilde{\Phi}|Y(t)|+Y^{T}(t-\tau(t)) G^{2} Y(t-\tau(t)) \\
& \leqslant-\tilde{a} \widetilde{V}(t)+b[\widetilde{V}(t)]_{\tau}
\end{aligned}
$$

where $\widetilde{A}(Y(t))=\operatorname{diag}\left(\tilde{a}_{1}\left(y_{1}(t)\right), \cdots, \tilde{a}_{n}\left(y_{n}(t)\right)\right), \widetilde{B}(Y(t))=\left(\tilde{b}_{1}\left(y_{1}(t)\right), \cdots, \tilde{b}_{n}\left(y_{n}(t)\right)\right)^{T}, \widetilde{F}(Y(t))=\left(\tilde{f}_{1}\left(y_{1}(t)\right), \cdots, \tilde{f}_{n}\left(y_{n}(t)\right)\right)^{T}$, $\widetilde{G}(Y(t))=\left(\tilde{g}_{1}\left(y_{1}(t)\right), \cdots, \tilde{g}_{n}\left(y_{n}(t)\right)\right)^{T}, \widetilde{G}(Y(t-\tau(t)))=\left(\tilde{g}_{1}\left(y_{1}\left(t-\tau_{1}(t)\right)\right), \cdots, \tilde{g}_{n}\left(y_{n}\left(t-\tau_{n}(t)\right)\right)\right)^{T}$.

When $t=t_{k}$, using the similar methods in the proof of [2, Theorem 3.1] results in that

$$
\widetilde{V}\left(t_{k}\right)=Y^{T}\left(t_{k}\right) P Y\left(t_{k}\right) \leqslant 2 \frac{\lambda_{\max }(P M P)}{\lambda_{\min } P} \widetilde{V}\left(t_{k}^{-}\right)+2 \frac{\lambda_{\max }\left(H N^{T} P N H\right)}{\lambda_{\min } P}\left[\widetilde{V}\left(t_{k}^{-}\right)\right]_{\tau} .
$$


Now it follows from $(\widetilde{C} 1),(\widetilde{C} 2),(\mathrm{C} 3)$ and [2, Lemma 2.2] that

$$
\widetilde{V}(t) \leqslant \rho[\widetilde{V}(0)]_{\tau} e^{-\left(\lambda-\frac{\ln \left(\rho \rho^{\lambda \tau}\right)}{\delta \tau}\right) t}, \quad t \geqslant 0
$$

or

$$
\sqrt{\left(u(t)-u^{*}\right)^{T}\left(u(t)-u^{*}\right)}=\sqrt{Y^{T}(t) Y(t)} \leqslant \sqrt{\frac{\rho \lambda_{\max } P}{\lambda_{\min } P}} \sqrt{\left[Y^{T}(0) Y(0)\right]_{\tau}} e^{-\frac{1}{2}\left(\lambda-\frac{\ln \left(\rho \rho^{\lambda \tau}\right)}{\delta \tau}\right) t}, \quad t \geqslant 0,
$$

which has proved that the equilibrium point $u^{*}$ of system (3.20) is globally exponentially stable with convergence rate $\frac{1}{2}\left(\lambda-\frac{\ln \left(\rho e^{\lambda \tau}\right)}{\delta \tau}\right)$.

Finally, we shall prove the second conclusion of Theorem 3.3.

Indeed, let $Y(t, x)=u(t, x)-u^{*}(x)$, where the stationary solution $u^{*}(x)=\left(u_{1}^{*}(x), \cdots, u_{n}^{*}(x)\right)^{T}$, then the system (3.19) can be transformed into

$$
\left\{\begin{array}{l}
\frac{\partial y_{i}(t, x)}{\partial t}=r_{i} \Delta y_{i}(t, x)-\tilde{a}_{i}\left(y_{i}(t, x)\right)\left[\tilde{b}_{i}\left(y_{i}(t, x)\right)-\sum_{j=1}^{n} c_{i j} \tilde{f}_{j}\left(y_{j}(t, x)\right)-\sum_{j=1}^{n} d_{i j} \tilde{g}_{j}\left(y_{j}\left(t-\tau_{j}(t), x\right)\right)\right], \quad t \geqslant 0, t \neq t_{k} \\
y_{i}\left(t^{+}, x\right)=m_{i} y_{i}\left(t^{-}, x\right)+\sum_{j=1}^{n} n_{i j} \tilde{h}_{j}\left(y_{j}\left(t^{-}-\tau_{j}(t), x\right)\right), \quad t=t_{k}, 0 \leqslant \tau_{j}(t) \leqslant \tau_{j}, \forall j \\
y_{i}(t, x)=0, \quad t \geqslant 0, x \in \partial \Omega, i=1,2, \cdots, n, \\
y_{i}(s, x)=\phi_{i}(s, x)-u_{i}^{*}(x), \quad-\tau \leqslant s \leqslant 0, \tau=\max _{1 \leqslant j \leqslant n} \tau_{j},
\end{array}\right.
$$

where $y_{i}, \tilde{a}_{i}, \tilde{b}_{i}, \tilde{f}_{j}, \tilde{g}_{j}$ and $\tilde{h}_{i}$ all are defined as those of [2].

$$
\int_{\Omega}\left|\nabla u_{i}(t, x)\right|^{2} d x \geqslant \lambda_{1} \int_{\Omega} u_{i}^{2}(t, x) d x
$$

On the other hand, the Poincare inequality and the Dirichlet zero boundary value yields

$$
\begin{aligned}
\int_{\Omega} Y^{T}(t, x) P R \Delta Y(t, x) d x & =-\int_{\Omega} \sum_{i=1}^{n} p_{i} r_{i} \sum_{j=1}^{m}\left(\frac{\partial y_{i}}{\partial x_{j}}\right)^{2} d x \\
& \leqslant-\lambda_{1} \int_{\Omega} \sum_{i=1}^{n} p_{i} r_{i} y_{i}^{2}(t, x) d x \leqslant 0,
\end{aligned}
$$

where $\lambda_{1}$ is the smallest positive eigenvalue of the following eigenvalue problem:

$$
\left\{\begin{aligned}
-\Delta \varphi(x) & =\lambda \varphi(x), \quad x \in \Omega \subset \mathbb{R}^{m}, \\
\varphi(x) & =0, \quad x \in \partial \Omega .
\end{aligned}\right.
$$

Constructing the Lyapunov functional as follows,

$$
\mathcal{V}(t)=\int_{\Omega} Y^{T}(t, x) P Y(t, x) d x=\int_{\Omega}\left|Y^{T}(t, x)\right| P|Y(t, x)| d x
$$


For the case of $t \neq t_{k}$, we compute the Dini derivative of $\mathcal{V}(t)$ alongside with the trajectories of (3.24),

$$
\begin{aligned}
D^{+} \mathcal{V}(t) & =\int_{\Omega}\left[-2 Y^{T}(t, x) P \widetilde{A}(Y(t, x)) \widetilde{B}(Y(t, x))+2 Y^{T}(t, x) P \widetilde{A}(Y(t, x)) C \widetilde{F}(Y(t, x))+2 Y^{T}(t, x) P \widetilde{A}(Y(t, x)) D \widetilde{G}(Y(t-\tau(t), x))\right] d x \\
& \leqslant \int_{\Omega}\left[-\left|Y^{T}(t, x)\right| \widetilde{\Phi}|Y(t, x)|+Y^{T}(t-\tau(t), x) G^{2} Y(t-\tau(t), x)\right] d x \\
& \leqslant \int_{\Omega}\left[-\tilde{a} \mathcal{V}(t, x)+b[\mathcal{V}(t, x)]_{\tau}\right] d x
\end{aligned}
$$

where $\widetilde{A}, \widetilde{B}, \widetilde{F}, \widetilde{G}$ all are defined as those of [2]. Completely similar as the proof of the first conclusion of Theorem 3.3 , we can also obtain

$$
\mathcal{V}\left(t_{k}\right)=\int_{\Omega} Y^{T}\left(t_{k}, x\right) P Y\left(t_{k}, x\right) d x \leqslant 2 \frac{\lambda_{\max }(P M P)}{\lambda_{\min } P} \mathcal{V}\left(t_{k}^{-}\right)+2 \frac{\lambda_{\max }\left(H N^{T} P N H\right)}{\lambda_{\min } P}\left[\mathcal{V}\left(t_{k}^{-}\right)\right]_{\tau},
$$

and

$$
\mathcal{V}(t) \leqslant \rho[\mathcal{V}(0)]_{\tau} e^{-\left(\lambda-\frac{\ln \left(\rho e^{\lambda \tau}\right)}{\delta \tau}\right) t}, \quad t \geqslant 0
$$

or

$$
\sqrt{\int_{\Omega}\left(u(t, x)-u^{*}\right)^{T}\left(u(t, x)-u^{*}\right) d x} \leqslant \sqrt{\frac{\rho \lambda_{\max } P}{\lambda_{\min } P}} \sqrt{\left[\int_{\Omega} Y^{T}(0, x) Y(0, x) d x\right]_{\tau}} e^{-\frac{1}{2}\left(\lambda-\frac{\ln \left(\rho e^{\lambda \tau}\right)}{\delta \tau}\right) t}, \quad t \geqslant 0,
$$

which has proved that the equilibrium point $u^{*}$ of system (3.20) is globally exponentially stable with convergence rate $\frac{1}{2}\left(\lambda-\frac{\ln \left(\rho e^{\lambda \tau}\right)}{\delta \tau}\right)$.

In fact, due to (3.25), the globally exponential stability of the equilibrium point $u^{*}$ of system (3.20) directly yields that the equilibrium point $u^{*}(x)$ of system (3.19) is also globally exponentially stable with the same convergence rate $\frac{1}{2}\left(\lambda-\frac{\ln \left(\rho e^{\lambda \tau}\right)}{\delta \tau}\right)$, which implies that the diffusion promotes the stability. The proof is completed.

Remark 4. Obviously, [2, Theorem 3.1] is the direct corollary of the conclusion (1) of Theorem 3.3 due to the Poincare inequality. In fact, the condition (C1) of [2, Theorem 3.1] is as follows,

$$
\Psi=\widetilde{\Psi}+\left(\begin{array}{ccc}
-2 l P R & 0 & 0 \\
0 & 0 & 0 \\
0 & 0 & 0
\end{array}\right)<0
$$

and the condition $(\mathrm{C} 2)$ of [2, Theorem 3.1] is as follows,

$$
a=\frac{\lambda_{\min } \Phi}{\lambda_{\max } P} \geqslant \tilde{a}=\frac{\lambda_{\min } \widetilde{\Phi}}{\lambda_{\max } P}>\frac{\lambda_{\max } G^{2}}{\lambda_{\min } P}=b \geqslant 0,
$$

where

$$
\Phi=2 l P R+\widetilde{\Phi}>0
$$

Remark 5. Conclusion (1) of Theorem 3.3 involved in the ordinary differential system (3.20) is completely similar as [2, Theorem 3.1] of the reaction-diffusion system (3.19). Due to Poincare inequality, [2, Theorem 3.1] becomes 
actually a corollary of the Conclusion (1) of Theorem 3.3, which implies that diffusions only promote the stability of the reaction-diffusion system. Actually, [2, Theorem 3.1] does not illuminate any negative effects on the stability of the reaction-diffusion system, compared with the conclusion (1) of Theorem 3.3. However, Conclusion (2) of Theorem 3.3 can show the two sides of the influence of diffusion on judging the stability of the diffusion system (3.19), for the existence of the solution $u^{*}(x)$ of the equations (3.22) may place more restrictions on the system, which may be similar as the condition (A2) of Theorem 3.1.

Remark 6. Particularly let $a_{i}\left(u_{i}\right) \equiv 1$ and $b_{i}\left(u_{i}\right)=b_{i} u_{i}$ with $b_{i} \in \mathbb{R}$ in the delayed reaction-diffusion Cohen-Grossberg neural networks (3.19), then the Cohen-Grossberg neural networks (3.19) is reduced to the following cellular neural networks

$$
\left\{\begin{array}{l}
\frac{\partial u_{i}(t, x)}{\partial t}=r_{i} \Delta u_{i}(t, x)-b_{i} u_{i}(t, x)+\sum_{j=1}^{n} c_{i j} f_{j}\left(u_{j}(t, x)\right)+\sum_{j=1}^{n} d_{i j} g_{j}\left(u_{j}\left(t-\tau_{j}(t), x\right)\right)-I_{i}, \quad t \geqslant 0, t \neq t_{k}, \\
u_{i}\left(t^{+}, x\right)=m_{i} u_{i}\left(t^{-}, x\right)+\sum_{j=1}^{n} n_{i j} h_{j}\left(u_{j}\left(t^{-}-\tau_{j}(t), x\right)\right), \quad t=t_{k}, 0 \leqslant \tau_{j}(t) \leqslant \tau_{j}, \forall j, \\
u_{i}(t, x)=0, \quad t \geqslant 0, x \in \partial \Omega, i=1,2, \cdots, n, \\
u_{i}(s, x)=\phi_{i}(s, x), \quad-\tau \leqslant s \leqslant 0, \tau=\max _{1 \leqslant j \leqslant n} \tau_{j} .
\end{array}\right.
$$

So the conclusions of Theorem 3.3 include the case of cellular neural networks. But if there is not impulse control in Theorem 3.3, the unique existence of the stationary solution of reaction-diffusion system should be given so that the global stability can be guaranteed for the reaction-diffusion system.

The following corollary can be derived by our Theorem 3.2 and Theorem 3.3.

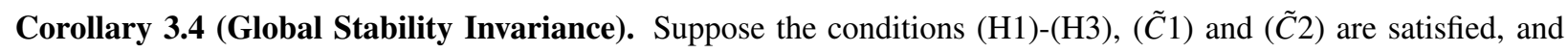
$a_{i}\left(u_{i}\right) \equiv 1, b_{i}\left(u_{i}\right)=b_{i} u_{i}, f_{i}\left(u_{i}\right)=g_{i}\left(u_{i}\right)$ as said in Remark 6. Besides, $H_{i} \equiv 0, m_{i} \equiv 1, n_{i j} \equiv 0, \underline{A}=\bar{A}=I$.

then we have the conclusions:

Conclusion (1) the constant equilibrium point $u^{*}$ of the following system is globally exponentially stable:

$$
\left\{\begin{array}{c}
\frac{d u_{i}(t)}{d t}=-b_{i} u_{i}(t)+\sum_{j=1}^{n} c_{i j} g_{j}\left(u_{j}(t)\right)+\sum_{j=1}^{n} d_{i j} g_{j}\left(u_{j}\left(t-\tau_{j}(t)\right)\right)-I_{i}, \quad t \geqslant 0, \\
u_{i}(s)=\phi_{i}(s), \quad-\tau \leqslant s \leqslant 0, \quad \tau=\max _{1 \leqslant j \leqslant n} \tau_{j}, \quad i=1,2, \cdots, n .
\end{array}\right.
$$

Conclusion (2) If there exits a solution $u^{*}(x)\left(\neq u^{*}\right.$ in common cases $)$ of the following equations:

$$
\left\{\begin{aligned}
0 & =r_{i} \Delta u_{i}(x)-b_{i} u_{i}(x)+\sum_{j=1}^{n} c_{i j} g_{j}\left(u_{j}(x)\right)+\sum_{j=1}^{n} d_{i j} g_{j}\left(u_{j}(x)\right)-I_{i}, \quad x \in \Omega \\
u_{i}(x) & =0, \quad x \in \partial \Omega, i=1,2, \cdots, n,
\end{aligned}\right.
$$

and if, in addition, there exists a scalar $\varepsilon>0$ such that

$$
-B+\frac{p}{2}\left(\varepsilon^{-1} I+\varepsilon G^{2}\right)<\lambda_{1} R
$$


where the constant $p>0$ satisfying $p^{2} I \geqslant(C+D)^{T}(C+D)$, and $R=\operatorname{diag}\left(r_{1}, r_{2}, \cdots, r_{n}\right), B=\operatorname{diag}\left(b_{1}, b_{2}, \cdots, b_{n}\right), C=$ $\left(c_{i j}\right), D=\left(d_{i j}\right)$, then the globally exponential stability of the equilibrium point $u^{*}$ of the system (3.29) directly yields that the equilibrium point $u^{*}(x)$ of the following reaction-diffusion system is also globally exponentially stable (in the classical meaning):

$$
\left\{\begin{array}{l}
\frac{\partial u_{i}(t, x)}{\partial t}=r_{i} \Delta u_{i}(t, x)-b_{i} u_{i}(t, x)+\sum_{j=1}^{n} c_{i j} g_{j}\left(u_{j}(t, x)\right)+\sum_{j=1}^{n} d_{i j} g_{j}\left(u_{j}\left(t-\tau_{j}(t), x\right)\right)-I_{i}, \quad t \geqslant 0, x \in \Omega, \\
u_{i}(t, x)=0, \quad t \geqslant 0, x \in \partial \Omega, i=1,2, \cdots, n, \\
u_{i}(s, x)=\phi_{i}(s, x), \quad-\tau \leqslant s \leqslant 0, \tau=\max _{1 \leqslant j \leqslant n} \tau_{j} .
\end{array}\right.
$$

Remark 7. Corollary 3.4 illuminates the invariance of global stability in the meaning of Definition 1 . Without the impulse control, the unique existence of the stationary solution must be considered for the reaction-diffusion system. In the conclusion (2) of Corollary 3.4, the condition (3.31) is the condition (A3). Besides, the existence of the stationary solution $u^{*}(x)$ of the equations (3.30) might be guaranteed by some condition similarly as (A2). In common cases, $u^{*}(x)$ is not necessarily equal to $u^{*}$. In fact, if the constant vector $u^{*} \neq 0, u^{*}$ is not any stationary solutions of reaction-diffusion system under Dirichlet zero boundary value. Below, an example will be designed to show it (see Statement 1).

To illuminates the effectiveness of Corollary 3.4, the Global Stability Invariance, we may present the following simple example in the case of $n=1$.

Example 3.5. In Corollary 3.4, set $n=1$, and $B=2, C=0.01, D=0.01, I_{1}=0.1, g(u)=u$ and then the Lipschitz constant of $g$ is $G=1$. Let $u^{*}=\frac{0.1}{1.98}$ is the unique solution of the following equation:

$$
0=-2 u+0.01 u+0.01 u+0.1
$$

Moreover, it is easy to verify that the conclusion (1) of Corollary 3.4 holds due to the fact that the related conditions of Corollary 3.4 are satisfied. That is, the constant equilibrium point $u^{*}=\frac{0.1}{1.98}$ of the system (3.29) is globally exponentially stable.

Besides, set $R=0.1, p=0.02, \varepsilon=1$, then $-B+\frac{p}{2}\left(\varepsilon^{-1} I+\varepsilon G^{2}\right)<0<\lambda_{1} R$, where $I=1$. According to Corollary 3.4 , if $u^{*}(x)$ is a solution of the following equation

$$
0=0.1 \Delta u-2 u+0.01 u+0.01 u+0.1, x \in \Omega ; \quad u=0, \quad x \in \partial \Omega,
$$

whose solution is corresponding to the critical point of the following functional

$$
\chi(u)=\frac{1}{2} \int_{\Omega}|\nabla u|^{2} d x+\frac{1}{2} \int_{\Omega} 19.8 u^{2} d x-\int_{\Omega} u d x,
$$

then the unique stationary solution $u^{*}(x)$ of the system (3.30) is globally exponentially stable in the classical meaning. As the selected special example, we are willing to prove the existence of the stationary solution $u^{*}(x)$. In fact, 
$\chi \in C^{1}\left(H_{0}^{1}(\Omega), \mathbb{R}\right)$, and $\chi$ is coercive, for

$$
\chi(u)=\frac{1}{2} \int_{\Omega}|\nabla u|^{2} d x+\frac{1}{2} \int_{\Omega} 19.8 u^{2} d x-\int_{\Omega} u d x \geqslant \frac{1}{2}\|u\|^{2}-c^{*}\|u\| \rightarrow+\infty, \quad\|u\| \rightarrow \infty,
$$

where $\|\cdot\|$ is the norm of $H_{0}^{1}(\Omega)$ with $\|u\|^{2}=\int_{\Omega}|\nabla u|^{2} d x$, and $c^{*}>0$ is a constant. It is easy to prove that $\chi$ satisfy the Palais-Smale condition, and $\chi$ is bounded below (see the methods used in proof of Statement 2 below). And hence, $\chi$ can attain its global minimum, say, $\chi\left(u^{*}(x)\right)$ at the point $u^{*}(x)$, on $H_{0}^{1}(\Omega)$ (see, e.g. the proof of [49, Lemma 2.5]). Due to the condition (3.31), the unique stationary solution $u^{*}(x)$ of the system (3.30) is globally exponentially stable in the classical meaning. Particularly, $u^{*}(x) \neq \frac{0.1}{1.98}, x \in \Omega$, for $u^{*}=\frac{0.1}{1.98}$ is a non-zero constant (see Statement 1 for details).

Statement 1. Let $u^{*}$ be a non-zero constant equilibrium point of an ordinary differential system. Then $u^{*}$ is not any stationary solutions of its corresponding reaction-diffusion system under Dirichlet zero boundary value, or it must lead to a contradiction.

Proof. Consider the following cellular neural networks in the case of $n=1$ :

$$
\left\{\begin{aligned}
\frac{d x(t)}{d t} & =-C x(t)+A f(x(t))+B f(x(t-\tau(t)))+J, \quad t \geqslant 0 \\
x(s) & =\xi(s) \text { is bounded in }[-\tau, 0] .
\end{aligned}\right.
$$

and its corresponding reaction-diffusion system:

$$
\left\{\begin{aligned}
\frac{\partial u(t, x)}{\partial t} & =D \Delta u(t, x)-C u(t, x)+A f(u(t, x))+B f(u(t-\tau(t), x))+J, \quad(t, x) \in \mathbb{R}_{+} \times \Omega, \Omega=(0,1) \subset \mathbb{R}, \\
u(t, x) & =0, t \geqslant 0, x \in \partial \Omega, \\
u(s, x) & =\xi(s, x) \text { is bounded in }[-\tau, 0] \times(0,1) .
\end{aligned}\right.
$$

where $D=0.001, C=1.8, A=0.2, B=0.1, J=1.09$, and $f(s)=0.05(s-6)$ for $s \in \mathbb{R}^{1}$.

Direct computation derives that $x=\frac{1000}{1785}=\frac{200}{357}$ is the constant equilibrium point of the system (3.32). Obviously, $f$ is Lipschitz continuous function with Lipschitz constant $\bar{l}=0.05$. In [31, Theorem 1], let $p=1$ and $N=1$, then

$$
-\mu_{1}\left(-N C N^{-1}\right)-\bar{l}\|N A\|_{1}\left\|N^{-1}\right\|_{1}=1.8-0.05 \times 0.2>0.05 \times 0.1=\bar{l}\|N B\|_{1}\left\|N^{-1}\right\|_{1}>0,
$$

and hence [31, Theorem 1] results in that there is the unique constant equilibrium point $x^{*}=\frac{1000}{1785}=\frac{200}{357}$ of the ordinary differential system (3.32) is globally exponentially stable.

Next, direct computation illuminates that the stationary solutions of the system (3.33) satisfies the following equation:

$$
\left\{\begin{aligned}
\frac{d^{2} u(x)}{d x^{2}} & =1785 u(x)-1000, \quad x \in \Omega=(0,1) \\
u(0) & =u(1)=0 .
\end{aligned}\right.
$$

Now it is easy to verify that

$$
u_{*}(x)=\frac{200\left(e^{-\sqrt{1785}}-1\right)}{357\left(e^{\sqrt{1785}}-e^{-\sqrt{1785}}\right)} e^{\sqrt{1785} x}-\frac{200\left(e^{\sqrt{1785}}-1\right)}{357\left(e^{\sqrt{1785}}-e^{-\sqrt{1785}}\right)} e^{-\sqrt{1785} x}+\frac{200}{357}, \quad \forall x \in[0,1] .
$$


is a solution of the equation (3.34), and it is also a nontrivial stationary solution of the reaction-diffusion (3.33).

Below, by the proof by contradiction, we shall prove that $u^{*}$ is not the equilibrium point of the reaction-diffusion (3.33), where $u^{*}=u^{*}(x) \equiv \frac{1000}{1785}=\frac{200}{357}$ for all $x \in(0,1)$ with $u^{*}(0)=u^{*}(1)=0$.

Firstly, by direct computation, the system (3.33) becomes the following system:

$$
\left\{\begin{aligned}
\frac{\partial u(t, x)}{\partial t} & =0.001 \Delta u(t, x)-1.8 u(t, x)+0.01 u(t, x)+0.005 u(t-\tau(t), x)+1, \quad t \geqslant 0, x \in \Omega=(0,1), \\
u(t, 0) & =u(t, 1)=0 \\
u(s, x) & =\xi(s, x) \text { is bounded in }[-\tau, 0] \times(0,1)
\end{aligned}\right.
$$

which is equivalent to the following system via the transformation $y(t, x)=u(t, x)-u^{*}$ :

$$
\left\{\begin{aligned}
\frac{\partial y(t, x)}{\partial t} & =0.001 \Delta y(t, x)-1.8 y(t, x)+0.01 y(t, x)+0.005 y(t-\tau(t), x), \quad t \geqslant 0, x \in \Omega=(0,1), \\
y(t, 0) & =y(t, 1)=0 \\
y(s, x) & =\eta(s, x) \text { is bounded in }[-\tau, 0] \times(0,1),
\end{aligned}\right.
$$

Consider the Lyapunov functional: $V(t, y(t, x))=\int_{\Omega} y^{2}(t, x) d x$, then the derivative $\frac{d V}{d t}$ alongside with the trajectories of the system (3.36) yields

$$
\begin{aligned}
\frac{d V(t, y(t, x))}{d t} & =\int_{\Omega} 2 y(t, x)(0.001 \Delta y(t, x)-1.8 y(t, x)+0.01 y(t, x)+0.005 y(t-\tau(t), x)) d x \\
& \leqslant-\left(0.002 \pi^{2}+3.575\right) \int_{\Omega} y^{2}(t, x) d x+0.005 \int_{\Omega} y^{2}(t-\tau(t), x) d x \\
& =-a V(t, y(t, x))+b V(t, y(t-\tau(t), x))
\end{aligned}
$$

where $a=0.002 \pi^{2}+3.575, b=0.005$, satisfying $a>b>0$. By employing [46, Lemma 3] or the methods in the proof of [40, Theorem 3], we can derive that the zero solution of the system (3.43) is globally exponentially stable with the convergence rate $\frac{1}{2}$, where $\lambda>0$ is the unique solution of the equation $\lambda=a-b e^{\lambda \tau}$. That is, the constant equilibrium point $u^{*}$ of the reaction diffusion system (3.33) is globally exponentially stable, and so $u^{*}$ is the unique equilibrium point of the reaction-diffusion system. However, $u_{*}(x)$ defined as (3.33) is its another equilibrium point. This contradiction shows that $u^{*}$ can not be the stationary solution of the reaction-diffusion system.

Remark 8. In [2, Theorem 3.1], $u^{*}$ is a non-zero constant vector in common cases. But [2, Theorem 3.1] told us that $u^{*}$ is a stable equilibrium point of a reaction-diffusion system under Dirichlet zero boundary value. Now Statement 1 illuminates that it must lead to a contradiction. On the other hand, the conclusion of Theorem 3.3 points out that $u^{*}(x)$ a stable equilibrium point of the reaction-diffusion system under Dirichlet zero boundary value, but $u^{*}(x)$ is not equal to the non-zero constant vector $u^{*}$. Such errors also occur in many previous literature (see, e.g. $[2,17,37,38]$ and the related references therein). 
Remark 9. Of course, some suitable non-zero constant vectors can be the equilibrium points or stationary solutions of delayed reaction-diffusion systems under Neumann boundary value (see, e.g. [15, 16, 34-36, 39, 47]) though they can not be the equilibrium points or stationary solutions of delayed reaction-diffusion systems under Dirichlet zero boundary value.

In the proof of Statement $1, u^{*}$ is the unique equilibrium point of ordinary differential system with Lipschitz assumption on active function $f$. Now we want to know whether the number of equilibrium points changes under the influence of inevitable diffusions.

Consider the following cellular neural networks in the case of $n=1$,

$$
\frac{d x(t)}{d t}=-C x(t)+A f(x(t))+B f(x(t-\tau(t)))+J, \quad \text { and } x \in \mathbb{R}^{1},
$$

and its corresponding reaction-diffusion cellular neural networks

$$
\left\{\begin{aligned}
\frac{\partial u(t, x)}{\partial t} & =D \Delta u(t, x)-C u(t, x)+A f(u(t, x))+B f(u(t-\tau(t), x))+J, \quad \text { and } t \geqslant 0, x \in \Omega \\
u(t, x) & =0, \quad x \in \partial \Omega
\end{aligned}\right.
$$

where $\Omega$ is an open bounded domain in $\mathbb{R}^{3}$ with smooth boundary $\partial \Omega, D \in \mathbb{R}^{1}$ is the diffusion coefficient with $D>0$, and $C, A$ both are positive real numbers, $J=0, B=0$, the function $f$ is defined as follow,

$$
f(u)=\left\{\begin{array}{l}
\frac{3 D}{A} \mu_{1} u^{\frac{1}{3}}+\frac{2 D}{A} \mu_{1}, \quad u \leqslant-1 \\
\frac{D}{A} \mu_{1} u, \quad u \in[-1,1] \\
\frac{3 D}{A} \mu_{1} u^{\frac{1}{3}}-\frac{2 D}{A} \mu_{1}, \quad u \geqslant 1
\end{array}\right.
$$

Here, we denote by $\mu_{i}$ the $i$ th positive eigenvalue of the following eigenvalue problem :

$$
\left\{\begin{aligned}
-\Delta u(x)+\frac{C}{D} u(x) & =\mu u(x), x \in \Omega, \\
u(x) & =0, \quad u \in \partial \Omega,
\end{aligned}\right.
$$

then $\mu_{1}=\frac{C}{D}+\lambda_{1}$, and $\mu_{2}>\mu_{1}([33])$.

Statement 2. If zero solution is the global stable unique equilibrium point of ordinary differential system (3.37), then its corresponding reaction-diffusion system (3.38) owns zero solution and other stationary solutions which are at least two non-zero functions or infinitely many positive functions and negative functions.

Proof. Firstly, it is easy to see from $f(0)=0$ and $J=0$ that zero solution is also an equilibrium point of the system (3.38).

Besides, we claim that the system (3.38) owns other stationary solutions which are at least two non-zero functions or infinitely many positive functions and negative functions. 
In fact, we know from (3.39) that $\left|\frac{d f(u)}{d u}\right| \leqslant \frac{D}{A} \mu_{1}$ for all $u \in \mathbb{R}^{1}$, because

$$
\frac{d f(u)}{d u}= \begin{cases}\frac{D}{A} \mu_{1} u^{-\frac{2}{3}}, & u \leqslant-1 \\ \frac{D}{A} \mu_{1}, & u \in[-1,1] \\ \frac{D}{A} \mu_{1} u^{-\frac{2}{3}}, & u \geqslant 1\end{cases}
$$

And hence, $f$ is Lipschitz continuous as follow,

$$
|f(u)-f(v)| \leqslant \frac{D}{A} \mu_{1}|u-v|, \quad \forall u, v \in \mathbb{R}^{1} .
$$

And the definition of $f$ yields

$$
F(u)=\int_{0}^{u} f(s) d s= \begin{cases}\frac{9}{4} \frac{D}{A} \mu_{1} u^{\frac{4}{3}}+\frac{2 D}{A} \mu_{1} u+\frac{1}{4} \frac{D}{A} \mu_{1}, & u \leqslant-1 \\ \frac{1}{2} \frac{D}{A} \mu_{1} u^{2}, \quad u \in[-1,1] & \\ \frac{9}{4} \frac{D}{A} \mu_{1} u^{\frac{4}{3}}-\frac{2 D}{A} \mu_{1} u+\frac{1}{4} \frac{D}{A} \mu_{1}, & u \geqslant 1 .\end{cases}
$$

Besides, if $u(x)$ is the stationary solution of the system (3.38), $u(x)$ is a solution of the following equation:

$$
\left\{\begin{aligned}
0 & =D \Delta u(x)-C u(x)+A f(u(x)), \quad x \in \Omega, \\
u(x) & =0, \quad x \in \partial \Omega,
\end{aligned}\right.
$$

whose solution is corresponding to the critical point of the following function:

$$
\mathfrak{J}(u)=\frac{1}{2} \int_{\Omega}|\nabla u|^{2} d x+\frac{C}{2 D} \int_{\Omega} u^{2} d x-\frac{A}{D} \int_{\Omega} F(u) d x,
$$

where $F(u)$ is given by (3.43).

Denote $H=\left\{u \in W_{0}^{1,2}(\Omega), \int_{\Omega}|\nabla u|^{2} d x+\frac{C}{D} \int_{\Omega} u^{2} d x<\infty\right\}$, in which the inner product is presented as follows,

$$
\langle u, v\rangle=\int_{\Omega} \nabla u \nabla v d x+\frac{C}{D} \int_{\Omega} u v d x,
$$

and its induced norm is denote by $\|\cdot\|_{H}$. Obviously, $\mathfrak{J} \in C^{1}\left(H, \mathbb{R}^{1}\right)$.

Besides, we claim that the functional $\mathfrak{J}$ is bounded below. In fact, if it is not true, there must exist a sequence $\left\{u_{n}\right\}$ in $H$ such that $\mathfrak{J}\left(u_{n}\right) \rightarrow-\infty$ as $\left\|u_{n}\right\|_{H} \rightarrow \infty$. So there must exist a scalar $\mathfrak{P}>0$ such that $\mathfrak{J}\left(u_{n}\right) \leqslant \mathfrak{P}$ for all $n$. Set $\mathfrak{W}_{n}=u_{n} /\left\|u_{n}\right\|_{H}$, then $\left\|\mathfrak{W}_{n}\right\|_{H} \equiv 1$ for all $n$. Moreover, Sobolev embedding theorem tells us that there exists $\mathfrak{W} \in H$ such that $\mathfrak{W}_{n} \rightarrow \mathfrak{W}$ in $H, \mathfrak{W}_{n} \rightarrow \mathfrak{W}$ in $L^{q}(\Omega)$ with $2 \leqslant q<2^{*}$, and $\mathfrak{W}_{n}(x) \rightarrow \mathfrak{W}(x)$, a.e. $x \in \Omega$, where $2^{*}$ is the critical sobolev exponent. Here and below, a subsequence of $\left\{u_{n}\right\}$ is still denoted by $\left\{u_{n}\right\}$ for convenience.

By employing the similar methods used in the proof of $\left[49\right.$, Lemma 2.1], we claim that $\|\mathfrak{W}\|_{H}^{2}=\mu_{1}\|\mathfrak{W}\|_{L^{2}(\Omega)}^{2}$. 
In fact, on one hand, it follows by (3.43) that there is such a constant $M>1$ big enough that

$$
\begin{aligned}
\frac{\mathfrak{P}}{\left\|u_{n}\right\|_{H}^{2}} & \geqslant \frac{\mathfrak{J}\left(u_{n}\right)}{\left\|u_{n}\right\|_{H}^{2}}=\frac{1}{2}\left[\left\|\mathfrak{W}_{n}\right\|_{H}^{2}-\mu_{1}\left\|\mathfrak{W}_{n}\right\|_{L^{2}(\Omega)}^{2}\right]+\frac{\frac{1}{2} \mu_{1} \int_{\Omega} u_{n}^{2} d x-\frac{A}{D} \int_{\Omega} F\left(u_{n}\right) d x}{\left\|u_{n}\right\|_{H}^{2}} \\
& =\frac{1}{2}\left[\left\|\mathfrak{W}_{n}\right\|_{H}^{2}-\mu_{1}\left\|\mathfrak{W}_{n}\right\|_{L^{2}(\Omega)}^{2}\right]+\frac{\int_{\left|u_{n}\right| \leqslant 1}\left[\frac{1}{2} \mu_{1} u_{n}^{2}-\frac{A}{D} F\left(u_{n}\right)\right] d x}{\left\|u_{n}\right\|_{H}^{2}}+\frac{\int_{\left|u_{n}\right| \geqslant 1}\left[\frac{1}{2} \mu_{1} u_{n}^{2}-\frac{A}{D} F\left(u_{n}\right)\right] d x}{\left\|u_{n}\right\|_{H}^{2}} \\
& =\frac{1}{2}\left[\left\|\mathfrak{W}_{n}\right\|_{H}^{2}-\frac{1}{2} \mu_{1}\left\|\mathfrak{W}_{n}\right\|_{L^{2}(\Omega)}^{2}\right]+0+\frac{\int_{\left|u_{n}\right| \geqslant 1}\left[\frac{1}{2} \mu_{1} u_{n}^{2}-\frac{A}{D} F\left(u_{n}\right)\right] d x}{\left\|u_{n}\right\|_{H}^{2}} \\
& \geqslant \frac{1}{2}\left[\left\|\mathfrak{W}_{n}\right\|_{H}^{2}-\frac{1}{2} \mu_{1}\left\|\mathfrak{W}_{n}\right\|_{L^{2}(\Omega)}^{2}\right]+0+\frac{\int_{1 \leqslant\left|u_{n}\right| \leqslant M} k_{M} d x}{\left\|u_{n}\right\|_{H}^{2}}+0 .
\end{aligned}
$$

On the other hand,

$$
\left|\frac{\int_{1 \leqslant\left|u_{n}\right| \leqslant M} k_{M} d x}{\left\|u_{n}\right\|_{H}^{2}}\right| \leqslant \frac{\left|k_{M}\right| \cdot m e s \Omega}{\left\|u_{n}\right\|_{H}^{2}} \rightarrow 0, \quad n \rightarrow \infty .
$$

Hence,

$$
\underset{n \rightarrow \infty}{\limsup }\left\|\mathfrak{W}_{n}\right\|_{H}^{2}-\mu_{1}\|\mathfrak{W}\|_{L^{2}(\Omega)}^{2} \leqslant 0 \Rightarrow \underset{n \rightarrow \infty}{\limsup }\left\|\mathfrak{W}_{n}\right\|_{H}^{2} \leqslant \mu_{1}\|\mathfrak{W}\|_{L^{2}(\Omega)}^{2} \leqslant\|\mathfrak{W}\|_{H}^{2},
$$

which together with the weak lower semi-continuity of the norm yields

$$
\underset{n \rightarrow \infty}{\limsup }\left\|\mathfrak{W}_{n}\right\|_{H}^{2} \leqslant \mu_{1}\|\mathfrak{W}\|_{L^{2}(\Omega)}^{2} \leqslant\|\mathfrak{W}\|_{H}^{2} \leqslant \liminf _{n \rightarrow \infty}\left\|\mathfrak{W}_{n}\right\|_{H}^{2}
$$

This implies the strong convergence of $\left\{\mathfrak{W}_{n}\right\}$ in Hilbert space $H$, and hence $\|\mathfrak{W}\|_{H}=1$, and $\|\mathfrak{W}\|_{H}^{2}=\mu_{1}\|\mathfrak{W}\|_{L^{2}(\Omega)}^{2}$, which means that $\mathfrak{W}$ is the eigenfunction of the least positive eigenvalue $\mu_{1}$. Let $\varphi_{1}$ be the positive eigenfunction in the one-dimensional eigenfunction space of the least positive eigenvalue $\mu_{1}$ such that $\left\|\varphi_{1}\right\|_{H}=1$, and $\varphi_{1}(x)>0$ for all $x \in \Omega$, then $\mathfrak{W}= \pm \varphi_{1}$. Since $\mathfrak{W}_{n}(x) \rightarrow \mathfrak{W}(x)$, a.e. $x \in \Omega$, there exits $\Omega^{*} \subset \Omega$ such that $m e s\left(\Omega / \Omega^{*}\right)=0$ and

$$
\left|\mathfrak{W}_{n}(x)\right|=\frac{\left|u_{n}(x)\right|}{\left\|u_{n}\right\|_{H}} \rightarrow \varphi_{1}(x), \quad \forall x \in \Omega^{*},
$$

which implies $\left|u_{n}(x)\right| \rightarrow+\infty, \forall x \in \Omega^{*}$. Now, it follows by (3.43) that

$$
\begin{aligned}
\mathfrak{P} & \geqslant \mathfrak{J}\left(u_{n}\right)=\frac{1}{2} \int_{\Omega}\left|\nabla u_{n}\right|^{2} d x+\frac{1}{2} \frac{C}{D} \int_{\Omega} u_{n}^{2} d x-\frac{A}{D} \int_{\Omega} F\left(u_{n}\right) d x \\
& \geqslant \int_{\Omega}\left[\frac{1}{2} \mu_{1} u_{n}^{2}-F(u)\right] d x=\int_{\Omega / \Omega^{*}}\left[\frac{1}{2} \mu_{1} u_{n}^{2}-F(u)\right] d x+\int_{\Omega^{*}}\left[\frac{1}{2} \mu_{1} u_{n}^{2}-F(u)\right] d x \\
& =\int_{\Omega^{*}}\left[\frac{1}{2} \mu_{1} u_{n}^{2}-F(u)\right] d x \rightarrow+\infty, \quad n \rightarrow \infty,
\end{aligned}
$$

which means that $\mathfrak{J}$ is bounded below.

By employing the similar methods used in [50], we shall prove that $\mathfrak{J}$ satisfies the (PS) condition.

In fact, if $\left\{u_{n}\right\}$ satisfies $\mathfrak{J}\left(u_{n}\right) \rightarrow a,\left\|\mathfrak{J}^{\prime}\left(u_{n}\right)\right\|_{H^{*}} \rightarrow 0$, and $n$ is big enough, we see

$$
a+o(1)=\mathfrak{J}\left(u_{n}\right)=\frac{1}{2} \int_{\Omega}\left|\nabla u_{n}\right|^{2} d x+\frac{1}{2} \frac{C}{D} \int_{\Omega} u_{n}^{2} d x-\frac{A}{D} \int_{\Omega} F\left(u_{n}\right) d x
$$


and

$$
\left\langle\mathfrak{J}^{\prime}\left(u_{n}\right), u_{n}\right\rangle=\int_{\Omega}\left|\nabla u_{n}\right|^{2} d x+\frac{C}{D} \int_{\Omega} u_{n}^{2} d x-\frac{A}{D} \int_{\Omega} f\left(u_{n}\right) u_{n} d x .
$$

And hence,

$$
\frac{1}{6}\left\|u_{n}\right\|_{H}^{2}+\frac{A}{D} \int_{\Omega}\left[\frac{1}{3} f\left(u_{n}\right) u_{n}-F\left(u_{n}\right)\right] d x \leqslant a+o(1)+\frac{1}{3}\left\|\mathfrak{J}^{\prime}\left(u_{n}\right)\right\|_{H^{*}}\left\|u_{n}\right\|_{H}
$$

or

$$
\frac{1}{6}\left\|u_{n}\right\|_{H}^{2} \leqslant a+o(1)+\frac{1}{3}\left\|\mathfrak{J}^{\prime}\left(u_{n}\right)\right\|_{H^{*}}\left\|u_{n}\right\|_{H}+\frac{A}{D} \int_{\Omega}\left|\frac{1}{3} f\left(u_{n}\right) u_{n}-F\left(u_{n}\right)\right| d x .
$$

On the other hand, (3.43) and (3.39) yield that there exists a constant $c_{*}>0$ big enough, satisfying

$$
\left\{\begin{array}{l}
\left|\frac{1}{3} f(u) u-F(u)\right| \leqslant c_{*} u^{\frac{4}{3}}+c_{*}, \quad|u| \geqslant 1 ; \\
\left|\frac{1}{3} f(u) u-F(u)\right|=\left|-\frac{1}{6} \frac{D}{A} \mu_{1} u^{2}\right| \leqslant c_{*}, \quad u \in[-1,1]
\end{array}\right.
$$

or

$$
\left|\frac{1}{3} f(u) u-F(u)\right| \leqslant c_{*} u^{\frac{4}{3}}+c_{*}, \quad \forall u \in \mathbb{R}^{1},
$$

which together with (3.44), Holder inequality and Poincare inequality implies

$$
\frac{1}{6}\left\|u_{n}\right\|_{H}^{2} \leqslant a+o(1)+c_{*}\left\|u_{n}\right\|_{H}+\frac{c_{*} A}{D} \operatorname{mes}(\Omega)+\frac{A}{D} c_{*}^{2}\left\|u_{n}\right\|_{H}^{\frac{4}{3}} .
$$

And then $\left\{u_{n}\right\}$ is bounded in $H$ due to (3.45).

Due to the fact $\|u\|^{2} \leqslant\|u\|_{E}^{2} \leqslant c_{*}\|u\|$, we see, $\|\cdot\|$ and $\|\cdot\|_{H}$ are a pair of equivalent norms. Moreover, we know from (3.39) that $f(u)$ satisfies the Caratheodory condition, and

$$
|f(u)| \leqslant c_{*}+c_{*}|u|^{\frac{1}{3}}, \quad \text { and } 0<\frac{1}{3}<2^{*}-1,
$$

which means that the bounded set $\left\{u_{n}\right\}$ with the condition $\left\|\mathfrak{J}^{\prime}\left(u_{n}\right)\right\|_{H^{*}} \rightarrow 0$ is a compact set in the Hilbert space $H$. This have verified that $\mathfrak{J}$ satisfies the (PS) condition.

On the one hand, if $\inf _{H} \mathfrak{J} \geqslant 0$, we claim that there are infinitely many positive stationary solutions and infinitely many negative stationary solutions for the reaction diffusion system (3.43) .

Indeed, since $\|\cdot\|$ and $\|\cdot\|_{H}$ are a pair of equivalent norms, Sobolev space $H$ ([43-45]) has the orthogonal decomposition $H=E\left(\mu_{1}\right) \oplus E\left(\mu_{1}\right)^{\perp}$, where $E\left(\mu_{k}\right)$ represents the eigenfunction space of $\mu_{k}$, and $E\left(\mu_{1}\right)^{\perp}=E\left(\mu_{2}\right) \oplus E\left(\mu_{3}\right) \oplus \cdots$. Obviously, $\mathfrak{J}$ satisfies (P1). In fact, if $u \in E\left(\mu_{1}\right)$ with $\|u\|_{H} \leqslant \delta$, the equivalence of norms in each finite dimensional space yields

$$
\|u\|_{H} \leqslant \delta \Rightarrow \int_{\Omega}|u(x)| d x<\delta_{1} \Rightarrow|u(x)|<1, \text { a.e. } x \in \Omega .
$$

where the positive number $\delta$ is small enough, and so is $\delta_{1}$. And then

$$
\begin{aligned}
\mathfrak{J}(u) & =\frac{1}{2}\|u\|_{H}^{2}-\frac{A}{D} \int_{\Omega} F(u) d x \\
& =\frac{1}{2}\|u\|_{H}^{2}-\mu_{1} \int_{\Omega} \frac{u^{2}}{2} d x=0 \leqslant 0, \quad u \in E\left(\mu_{1}\right),\|u\|_{H} \leqslant \delta .
\end{aligned}
$$


which together with $\inf _{H} \mathfrak{J} \geqslant 0$ implies that all $u \in E\left(\mu_{1}\right)$ with $\|u\|_{H} \leqslant \delta$ are the stationary solutions of the reaction diffusion system (3.38). Moreover, $E\left(\mu_{1}\right)$ implies that there are infinitely many positive stationary solutions and infinitely many negative stationary solutions for the reaction diffusion system (3.38).

On the other hand, if $\inf _{H} \mathfrak{J}<0$, we see, the condition (P3) holds. Then we claim that there are at least three equilibrium solutions including two non-zero stationary solutions, for the reaction diffusion system (3.38) if $\inf _{H} \mathfrak{J}<$ 0 .

In fact, (3.46) implies that (P1) holds. In order to apply Lemma 2.2, we only need to verify the condition (P2) of Lemma 2.2. Next, for $u \in E\left(\mu_{1}\right)^{\perp}$, let $u=v+z$, where $v \in E\left(\mu_{2}\right), z \in E\left(\mu_{3}\right) \oplus E\left(\mu_{4}\right) \oplus \cdots$. Then we get

$$
\begin{aligned}
\mathfrak{J}(u) & \geqslant \frac{1}{2}\left(\mu_{2} \int_{\Omega} v^{2} d x+\mu_{2} \int_{\Omega} z^{2} d x\right)+\frac{1}{2}\left[\|z\|_{H}^{2}-\mu_{2} \int_{\Omega} z^{2} d x\right]-\frac{A}{D} \int_{\Omega} F(u) d x \\
& \geqslant \frac{\mu_{2}}{2}\left(\int_{\Omega} v^{2} d x+\int_{\Omega} z^{2} d x\right)+\frac{1}{2}\left(1-\frac{\mu_{2}}{\mu_{3}}\right)\|z\|_{H}^{2}-\frac{A}{D} \int_{\Omega} F(u) d x \\
& =\left[\frac{1}{2} \mu_{2} \int_{\Omega} u^{2} d x-\frac{A}{D} \int_{\Omega} F(u) d x\right]+\frac{1}{2}\left(1-\frac{\mu_{2}}{\mu_{3}}\right)\|z\|_{H}^{2}, \quad \forall u \in E\left(\mu_{1}\right)^{\perp} .
\end{aligned}
$$

Due to (3.43), there exists $\delta \in(0,1)$ such that

$$
\frac{2 A}{D} F(u)=\mu_{1} u^{2} \leqslant \mu_{2} u^{2}, \quad \text { if }|u| \leqslant \delta<1, u \in E\left(\mu_{1}\right)^{\perp} .
$$

Moreover, for this $\delta$, there exists correspondingly $\delta_{2}>0$ such that for $u \in E\left(\mu_{2}\right)$ with $\|u\|_{H} \leqslant \delta_{2}$, we get $|u(x)| \leqslant \frac{\delta}{2}$, a.e. $x \in \Omega$ in view of the equivalence of norms in finite dimensional space.

Define

$$
\Omega_{1}=\{x \in \Omega:|u(x)| \leqslant \delta\}, \quad \Omega_{2}=\{x \in \Omega:|u(x)|>\delta\} .
$$

Due to the orthogonal decomposition of the Sobolev space $H$ and $u=v+z$, we see, $\|u\|_{H} \leqslant \delta_{2} \Rightarrow\|v\|_{H} \leqslant \delta_{2}$, which implies

$$
|v(x)| \leqslant \frac{\delta}{2} \leqslant \frac{1}{2}|u(x)|, \quad|z(x)| \geqslant|u(x)|-|v(x)| \geqslant \frac{1}{2}|u(x)|, \text { a.e. } x \in \Omega_{2} .
$$

Besides, (3.43) yields

$$
\left|\frac{1}{2} \mu_{2} u^{2} d x-\frac{A}{D} F(u)\right| \leqslant c_{*}|u|^{3} \leqslant 8 c_{*}|z|^{3}, x \in \Omega_{2}
$$

So we can see it from the orthogonal decomposition of the Sobolev space $H$ and the Sobolev embedding theorem that for all $u \in E\left(\mu_{1}\right)^{\perp}$, if $\|u\|_{H} \leqslant \delta_{2}$, we get

$$
\begin{aligned}
\mathfrak{J}(u) & \geqslant\left[\frac{1}{2} \mu_{2} \int_{\Omega_{1}} u^{2} d x-\frac{A}{D} \int_{\Omega_{1}} F(u) d x\right]+\frac{1}{2}\left(1-\frac{\mu_{2}}{\mu_{3}}\right)\|z\|_{H}^{2}+\left[\frac{1}{2} \mu_{2} \int_{\Omega_{2}} u^{2} d x-\frac{A}{D} \int_{\Omega_{2}} F(u) d x\right] \\
& \geqslant \frac{1}{2}\left(1-\frac{\mu_{2}}{\mu_{3}}\right)\|z\|_{H}^{2}-8 c_{*} \int_{\Omega_{2}}|z|^{3} d x \\
& \geqslant \frac{1}{2}\left(1-\frac{\mu_{2}}{\mu_{3}}\right)\|z\|_{H}^{2}-8 c_{*}^{2}\|z\|_{H}^{3},
\end{aligned}
$$

which implies that the condition (P2) holds. And now all the conditions of Saddle point theorem are satisfied. According to Lemma 2.2, we have proved the claim. And the proof is completed. 
Remark 10. Statement 2 actually illuminates that under Lipschtiz assumption on active function, diffusion may make an equilibrium point become infinitely many stationary solution. The deeper purpose of Statement 2 may be revealed in the final section for further considerations (see Problem 1). On the other hand, according to the Introduction in [51], the function $f$ defined by (3.39) satisfies the conditions [51, (7)] and [51, (8)], and hence, the zero solution is actually the unique equilibrium point of the ordinary differential system (3.37). Thereby, Statement 2 has actually verified that under the Lipschitz assumption on the function $f$, the small diffusion can truly make one equilibrium point become multiple stationary solutions (three stationary solutions, even infinitely many stationary solutions).

\section{Numerical example}

Example 4.1. Consider the following switched financial system with $\sigma \in\{1,2,3\}$,

$$
\begin{aligned}
& \left\{\begin{array}{l}
\frac{\partial y(t, x)}{\partial t}=D_{1} \Delta y(t, x)-C_{1} y(t, x)+A_{1} g(y(t, x))+B_{1} g(y(t-\tau(t), x))+J_{1}, \quad(t, x) \in \mathbb{R}_{+} \times \Omega_{1}, \\
y_{i}(t, x)=0, t \geqslant 0, x \in \partial \Omega_{1}, i=1,2,
\end{array}\right. \\
& \left\{\begin{array}{l}
\frac{\partial y(t, x)}{\partial t}=D_{2} \Delta y(t, x)-C_{2} y(t, x)+A_{2} g(y(t, x))+B_{2} g(y(t-\tau(t), x))+J_{2}, \quad(t, x) \in \mathbb{R}_{+} \times \Omega_{2}, \\
y_{i}(t, x)=0, t \geqslant 0, x \in \partial \Omega_{2}, i=1,2,
\end{array}\right.
\end{aligned}
$$

and

$$
\left\{\begin{array}{l}
\frac{\partial y(t, x)}{\partial t}=D_{3} \Delta y(t, x)-C_{3} y(t, x)+A_{3} g(y(t, x))+B_{3} g(y(t-\tau(t), x))+J_{3}, \quad(t, x) \in \mathbb{R}_{+} \times \Omega_{3}, \\
y_{i}(t, x)=0, t \geqslant 0, x \in \partial \Omega_{3}, i=1,2
\end{array}\right.
$$

or the following corresponding homogeneous equations:

$$
\left\{\begin{array}{l}
\frac{\partial u(t, x)}{\partial t}=D_{\sigma} \Delta u(t, x)-C_{\sigma} u(t, x)+A_{\sigma} f(u(t, x))+B_{\sigma} f(u(t-\tau(t), x)), \quad(t, x) \in \mathbb{R}_{+} \times \Omega_{\sigma}, \\
u_{i}(t, x)=0, t \geqslant 0, x \in \partial \Omega_{\sigma}, i=1,2 ; \quad \sigma \in\{1,2,3\}
\end{array}\right.
$$

equipped with the initial value:

$u_{j}(s, x)=\phi_{i}(s, x)=\prod_{\sigma=1}^{3} \sin ^{j}\left[x_{1}^{33}\left(x_{1}-5(\sigma+1)\right)^{353} x_{2}^{63}\left(x_{2}-5(\sigma+1)\right)^{79}\right], j=1,2,-\tau \leqslant s \leqslant 0, x \in \Omega_{\sigma}, \sigma \in\{1,2,3\}$,

where $\Omega_{1}=[0,1] \times[0,1], \Omega_{2}=[0,1.3] \times[0,1.3], \Omega_{3}=[0,1.5] \times[0,1.5], \lambda_{11}=19.7392, \lambda_{21}=11.68, \lambda_{31}=8.7730($ see Remark 13).

$$
\text { Set } c=100000000, I=\operatorname{diag}(1,1) \text {, and }
$$




$$
\begin{gathered}
C_{1}=\left(\begin{array}{cc}
0.448 & 0 \\
0 & 0.441
\end{array}\right), C_{2}=\left(\begin{array}{cc}
0.455 & 0 \\
0 & 0.441
\end{array}\right), C_{3}=\left(\begin{array}{cc}
0.438 & 0 \\
0 & 0.433
\end{array}\right), \\
A_{1}=\left(\begin{array}{cc}
0.45 & 0.00003 \\
-0.00003 & 0.44
\end{array}\right), A_{2}=\left(\begin{array}{cc}
0.452 & 0.00001 \\
-0.00001 & 0.441
\end{array}\right), A_{3}=\left(\begin{array}{cc}
0.439 & 0.000015 \\
-0.00001 & 0.433
\end{array}\right), \\
B_{1}=\left(\begin{array}{cc}
0.446 & -0.00003 \\
0.00003 & 0.442
\end{array}\right), B_{2}=\left(\begin{array}{cc}
0.458 & -0.00001 \\
0.00001 & 0.441
\end{array}\right), B_{3}=\left(\begin{array}{cc}
0.437 & -0.000015 \\
0.00001 & 0.433
\end{array}\right),
\end{gathered}
$$

Let $g_{i}\left(y_{i}\right)=\frac{39+2 y_{i}+0.000001 \sin y_{i}}{4}$, and then $G=\operatorname{diag}(0.51,0.51)$. Set $J_{\sigma}=(0.2 \sin \sigma,-0.1 \cos \sigma)^{T}, \sigma=1,2,3$, then the direct calculation can verify that both conditions (A1) and (A2) hold.

For example, in the case of $\sigma=1$, direct computation derives

$$
0 \leqslant\left[-C_{1} v+A_{1} g(v)+B_{1} g(v)+J_{1}\right]=\left(\begin{array}{l}
0.448 \times \frac{39+0.000001 \sin v_{1}}{2} \\
0.441 \times \frac{39+0.000001 \sin v_{2}}{2}
\end{array}\right)+\left(\begin{array}{c}
0.2 \sin 1 \\
-0.1 \cos 1
\end{array}\right) \leqslant c D_{1} E,
$$

which means that (A2) holds in the case of $\sigma=1$. Similarly we can compute and verify that (A2) holds in the case of $\sigma=2,3$.

\section{Case 1}

Set

$$
D_{1}=\left(\begin{array}{cc}
0.05 & 0 \\
0 & 0.055
\end{array}\right), D_{2}=\left(\begin{array}{cc}
0.07 & 0 \\
0 & 0.075
\end{array}\right), D_{3}=\left(\begin{array}{cc}
0.09 & 0 \\
0 & 0.095
\end{array}\right),
$$

Moreover, in (3.17), let $p_{\sigma} \equiv 1$, then it is easy to verify that $p_{\sigma}>0$ satisfies $p_{\sigma}^{2} I \geqslant\left(A_{\sigma}+B_{\sigma}\right)^{T}\left(A_{\sigma}+B_{\sigma}\right)$ for each $\sigma$. In addition, set $\varepsilon=2$,

$$
-C_{\sigma}+\frac{p_{\sigma}}{2}\left(\varepsilon^{-1} I+\varepsilon G^{2}\right)<0.1 I<\lambda_{\sigma 1} D_{\sigma}, \quad \text { for all } \sigma=1,2,3 .
$$

Then the condition (A3) holds (Below, it can be verified similarly that (A3) holds in Case 2-3, too).

Let $q=1.00001, \Psi=0.00018 I$ and $\tau=3.5$, then employing computer LMI toolbox to solve the inequality (3.1) derives the following feasible data:

$$
\beta_{1}=0.5676, \beta_{2}=0.3633, \beta_{3}=0.0691, \gamma=0.38
$$

then the switched delayed reaction-diffusion system (2.3) equipped with the initial value (2.4) is globally exponentially stable with the convergence rate $19 \%$ due to Theorem 3.2 .

\section{Case 2}

If replacing the diffusion coefficients (4.4) with the following diffusion coefficients

$$
D_{1}=\left(\begin{array}{cc}
0.1 & 0 \\
0 & 0.15
\end{array}\right), D_{2}=\left(\begin{array}{cc}
0.15 & 0 \\
0 & 0.2
\end{array}\right), D_{3}=\left(\begin{array}{cc}
0.1 & 0 \\
0 & 0.15
\end{array}\right),
$$


and other data of Case 1 are not changed, we can use the computer LMI toolbox to solve the inequality (3.1), resulting in the following feasible data:

$$
\beta_{1}=0.6769, \beta_{2}=0.2333, \beta_{3}=0.0898, \gamma=0.44
$$

then the switched delayed reaction-diffusion system (2.3) equipped with the initial value (2.4) is globally exponentially stable with the convergence rate $22 \%$ due to Theorem 3.2 .

\section{Case 3}

If replacing $\tau=3.5$ with $\tau=3$, and other data of Case 1 are not changed, we can use the computer LMI toolbox to solve the inequality (3.1), resulting in the following feasible data:

$$
\beta_{1}=0.6616, \beta_{2}=0.3113, \beta_{3}=0.0271, \gamma=0.58
$$

then the switched delayed reaction-diffusion system (2.3) equipped with the initial value (2.4) is globally exponentially stable with the convergence rate $29 \%$ due to Theorem 3.2 .

Table 1.Comparisons the influences on the convergence rate $\frac{\lambda}{2}$ under different diffusion coefficients with the same other data

\begin{tabular}{|c|c|c|}
\hline & Case 1 & Case 2 \\
\hline diffusion coefficient & $(4.4)$ (smaller) & (4.5) (bigger) \\
\hline Convergence rate & $19 \%$ & $22 \%$ \\
\hline
\end{tabular}

Remark 11. Table 1 tells us that the larger the diffusion coefficient, the faster the convergence rate. On the other hand, the harsh condition (A2) illuminates that the diffusion makes it more difficult to judge the stability of the system.

Table 2.Comparisons the influences on the convergence rate $\frac{\lambda}{2}$ under different upper limits of delays with the same other data

\begin{tabular}{|c|c|c|}
\hline & Case 1 & Case 3 \\
\hline diffusion coefficient & $\tau=3.5$ & $\tau=3$ \\
\hline Convergence rate & $19 \%$ & $29 \%$ \\
\hline
\end{tabular}

Remark 12. Table 2 indicates that the larger the upper bound $\tau$ of time delays, the slower the convergence speed $\frac{\gamma}{2}$.

Remark 13. (see,e.g.,[14-16]) The smallest positive eigenvalue of $-\Delta_{p}$ in $W_{0}^{1, p}(0, T)$ is

$$
\lambda_{1}=\left(\frac{2}{T} \int_{0}^{(p-1)^{\frac{1}{p}}} \frac{d t}{\left(1-\frac{t^{p}}{p-1}\right)^{\frac{1}{p}}}\right)^{p} .
$$

If $\Omega=\left\{\left(x_{1}, x_{2}\right)^{T}: 0<x_{1}<\alpha, 0<x_{2}<\beta\right\} \subset \mathbb{R}^{2}$ and $W_{0}^{1, p}(\Omega)$ with $p=2$, the first eigenvalue $\lambda_{1}=\left(\frac{\pi}{\alpha}\right)^{2}+\left(\frac{\pi}{\beta}\right)^{2}$.

Besides, it is well known that there is the following approximate substitution of Poincare inequality lemma :

Remark 14. Let $\Omega$ be a cube $\left|x_{i}\right|<l_{i}(i=1,2, \cdots, n)$ and let $\mu(x)$ be a real-valued function belonging to $C^{1}(\Omega)$ which vanish on the boundary $\partial \Omega$ of $\Omega$, i.e., $\left.\mu(x)\right|_{\partial \Omega}=0$, then

$$
\int_{\Omega} \mu^{2}(x) d x \leqslant l_{i}^{2} \int_{\Omega}\left|\frac{\partial \mu}{\partial x_{i}}\right|^{2} d x .
$$




\section{Conclusions and further considerations}

By constructing a compact operator on a convex set, the author makes up for the loss of compactness in infinite dimensional space. Using a fixed point theorem, variational methods and Lyapunov functional method results in the existence positive bounded stationary solution, which is exponentially stable. Moreover, by using the first positive eigenvalue of Laplace operator $-\Delta$ to restrain Lipschitz constants, the author proposes the uniqueness theorem of the stationary solution of reaction-diffusion system under Dirichlet zero boundary value, and thereby the stability of Theorem 3.1 becomes global. Not only that, Theorem 3.1 and Theorem 3.2 derive a corollary on the variance of global stability. Moreover, Statement 1 points out the fact that non-zero constant vector can not be a stationary solution of the reaction-diffusion system under Dirichlet zero boundary value. Besides, Statement 2 points out that the influence of diffusions changes the number of the system under Lipschitz assumptions on active functions. Finally, a numerical example is presented to illuminate the effectiveness of the proposed methods.

Below, some interesting problems are proposed as follows,

Problem 1. How to improve the example in Statement 2 or add a suitable condition to the example in Statement 2 so that the constant equilibrium point of the ordinary differential system can be truly proved to be globally asymptotically stable. At the same time, the corresponding reaction-diffusion system owns multiple stationary solutions. If so, many global stability results of delayed neural networks in the form of ordinary differential equations may only be locally asymptotical stability criteria in real engineering due to the inevitable diffusions.

Problem 2. How to replace (A2) with a weaker condition in Theorem 3.1 ?

Problem 3. Is the condition (A3) of Theorem 3.2 necessary for the uniqueness? If not, what's the weaker condition?

\section{Funding Statement}

The work is supported by the Application basic research project of science and Technology Department of Sichuan Province (No. 2020YJ0434) and the Major scientific research projects of Chengdu Normal University in 2019 (No. CS19ZDZ01)

\section{References}

[1] X. Lu, W. Chen, Z. Ruan, T. Huang, A new method for global stability analysis of delayed reaction-diffusion neural networks,Neurocomputing 317 (2018) 127-136.

[2] X. Zhang, S. Wu, K. Li, Delay-dependent exponential stability for impulsive Cohen-Grossberg neural networks with time-varying delays and reaction-diffusion terms, Commun. Nonlinear Sci. Numer. Simulat. 16 (2011) 1524-1532.

[3] T. Wei, P. Lin, Y. Wang, L. Wang, Stability of stochastic impulsive reaction-diffusion neural networks with S-type distributed delays and its application to image encryption, Neural Net. 116(2019) 35-45.

[4] Hongyong Zhao, Zisen Mao. Boundedness and stability of nonautonomous cellular neural networks with reaction-diffusion terms, Mathematics and Computers in SimulationVolume 79, Issue 5January 2009Pages 1603-1617

[5] Kai Wang, Zhidong Teng, Haijun Jiang. Global exponential synchronization in delayed reaction-diffusion cellular neural networks with the Dirichlet boundary conditions. Mathematical and Computer Modelling, 2010, 52, 12-24 
[6] Z. Yang, W. Zhou, T. Huang, Input-to-state stability of delayed reaction-diffusion neural networks with impulsive effects, Neurocomputing 33314(2019) 261-272.

[7] J. Hou, Y. Huang, E. Yang, $\psi$-type stability of reaction-diffusion neural networks with time-varying discrete delays and bounded distributed delays, Neurocomputing 3407 (2019) 281-293.

[8] P. Balasubramaniam, C. Vidhya. Global asymptotic stability of stochastic BAM neural networks with distributed delays and reactiondiffusion terms. Journal of Computational and Applied Mathematics 234 (2010) 3458-3466

[9] Zhilin Pu, Ruofeng Rao. Delay-dependent LMI-based robust stability criterion for discrete and distributed time-delays Markovian jumping reaction-diffusion CGNNs under Neumann boundary value NeurocomputingVolume 1711 January 2016Pages 1367-1374

[10] Ruofeng Rao, Shouming Zhong, Zhilin Pu. On the role of diffusion factors in stability analysis for p-Laplace dynamical equations involved to BAM Cohen-Grossberg neural network NeurocomputingVolume 2235 February 2017Pages 54-62

[11] Ruofeng Rao, Shouming Zhong. Input-to-state stability and no-inputs stabilization of delayed feedback chaotic financial system involved in open and closed economy. Discrete and Continuous Dynamical Systems, Series S, doi:10.3934/dcdss.2020280.

[12] W. Chen, Dynamics and control of a financial system with time-delayed feedbacks, Chaos Soli. Frac., 37 (2008), 1198-1207.

[13] D. Gilbarg, N.S. Trudinger, Elliptic partial differential equations of second order, Berlin, Springer, 1983.

[14] R. Rao, S. Zhong, X. Wang, Stochastic stability criteria with LMI conditions for Markovian jumping impulsive BAM neural networks with mode-dependent time-varying delays and nonlinear reaction-diffusion, Comm. Nonlinear Sci. Num. Simu. 19(2014) 258-273.

[15] Jie Pan, Shouming Zhong. Dynamical behaviors of impulsive reaction-diffusion Cohen-Grossberg neural network with delays. Neurocomputing, Volume 73, Issues 7-9March 2010Pages 1344-1351

[16] Jie Pan, Xinzhi Liu, Shouming Zhong. Stability criteria for impulsive reaction-diffusion Cohen-Grossberg neural networks with timevarying delays. Mathematical and Computer Modelling, Volume 51, Issues 9-10May 2010Pages 1037-1050

[17] J. Pan, S. Zhong. Dynamic Analysis of Stochastic Reaction-Diffusion Cohen-Grossberg Neural Networks with Delays. Advances in Difference Equations, 2009, Article ID 410823, 18 pages.

[18] Z. Guo, S. Ou, J. Wang, Multistability of switched neural networks with sigmoidal activation functions under state-dependent switching, Neural Net. 122(2020)239-252

[19] D. Yang, X. Li, S. Song. Design of State-Dependent Switching Laws for Stability of Switched Stochastic Neural Networks With TimeDelays, IEEE Trans. Neu. Net. Learn. Syst. 2019.

[20] C. Hsu, J. Lin, Stability of traveling wave solutions for nonlinear cellular neural networks with distributed delays, J. Math. Anal. Appl. 470(2019) 388-400.

[21] X. Li, X. Fu, Stability analysis of stochastic functional differential equations with infinite delay and its application to recurrent neural networks, J. Comput. Appl. Math. 234(2010) 407-417.

[22] L.O. Chua ; L. Yang. Cellular neural networks: theory. IEEE Transactions on Circuits and Systems, 1988, 35(10) : 1257-1272.

[23] L.O. Chua ; L. Yang. Cellular neural networks: applications. IEEE Transactions on Circuits and Systems, 1988,35(10) : 1273-1290.

[24] Chuangxia Huang, Renli Su, Jinde Cao, Songlin Xiao. Asymptotically stable high-order neutral cellular neural networks with proportional delays and D operators, Mathematics and Computers in Simulation, Volume 171May 2020Pages 127-135

[25] Huizhen Qu, Tianwei Zhang, Jianwen Zhou. Global stability analysis of S-asymptotically $\omega$-periodic oscillation in fractional-order cellular neural networks with time variable delays, Neurocomputing, In press, corrected proofAvailable online 10 March 2020

[26] Jinde Cao, Qiong Li. On the Exponential Stability and Periodic Solutions of Delayed Cellular Neural Networks, Journal of Mathematical Analysis and Applications, Volume 252, Issue 1, 1 December 2000, Pages 50-64

[27] Renwei Jia. Finite-time stability of a class of fuzzy cellular neural networks with multi-proportional delays, Fuzzy Sets and Systems, Volume 319, 15 July 2017, Pages 70-80

[28] Qiankun Song, Zidong Wang. Dynamical behaviors of fuzzy reaction-diffusion periodic cellular neural networks with variable coefficients and delays, Applied Mathematical Modelling, 33 (2009) 3533-3545

[29] Rakkiyappan, R., Dharani, S., Zhu, Q. (2015). Synchronization of reactiondiffusion neural networks with time-varying delays via stochas- 
tic sampleddata controller. Nonlinear Dynamics, 79(1), 485-500.

[30] M. Zhao and J. Wang, $H_{\infty}$ control of a chaotic finance system in the presence of external disturbance and input time-delay, Appl. Math. Comput., 233 (2014), 320-327.

[31] Qingshan Liu, Jinde Cao. Improved global exponential stability criteria of cellular neural networks with time-varying delays. Mathematical and Computer Modelling 43 (2006) 423-432.

[32] DEIMLING K. Nonlinear functional analysis. Berlin: Springer, 1985.

[33] Ashbaugh M S , Benguria R D . Optimal bounds for ratios of eigenvalues of one-dimensional Schrodinger operators with Dirichlet boundary conditions and positive potentials. Communications in Mathematical Physics, 1989, 124(3):403-415.

[34] Zuoan Li, Kelin Li. Stability analysis of impulsive Cohen-Grossberg neural networks with distributed delays and reaction-diffusion terms Applied Mathematical Modelling, 2009, 33(3), 1337-1348.

[35] Jinling Liang, Jinde Cao. Global exponential stability of reaction-diffusion recurrent neural networks with time-varying delays Physics Letters A, 314(5-6), 2003, 434-442.

[36] P. Balasubramaniam, C. Vidhya. Global asymptotic stability of stochastic BAM neural networks with distributed delays and reactiondiffusion terms. Journal of Computational and Applied Mathematics, 2010, 234(12), 3458-3466

[37] Ailong Wu, Chaojin Fu. Global exponential stability of non-autonomous FCNNs with Dirichlet boundary conditions and reactiondiffusion terms. Applied Mathematical Modelling, 2010, 34(10), 3022-3029

[38] Wan-Tong Li, Xiang-Ping Yan, Cun-Hua Zhang. Stability and Hopf bifurcation for a delayed cooperation diffusion system with Dirichlet boundary conditions Chaos, Solitons \& Fractals, 2008, 39,1538C1549.

[39] Kelin Li, Qiankun Song. Exponential stability of impulsive CohenCGrossberg neural networks with time-varying delays and reactiondiffusion terms. Neurocomputing, 72(2008)231C240.

[40] Ruofeng Rao, Jialin Huang, Shouming Zhong. Global exponential stability of reaction-diffusion BAM neural networks (In Chinese). J. Jilin Univ. (Sci. Ed.), 50 (2012), 1086-1090.

[41] Ruofeng Rao, Shouming Zhong. Existence of Exponential $p$-Stability Nonconstant Equilibrium of Markovian Jumping Nonlinear Diffusion Equations via Ekeland Variational Principle. Advances in Mathematical Physics Volume 2015, Article ID 812150, 10 pages

[42] Haim Brezis, Louis Nirenberg. Remarks on finding critical points. Communications on Pure \& Applied Mathematics, 44 (1991), 939-963.

[43] Xing-Ping Wu, Chun-Lei Tang. Remarks on existence and multiplicity of solutions for a class of semilinear elliptic equations. Journal of Mathematical Analysis and Applications, Volume 319, Issue 11 July 2006Pages 369-376

[44] Ruofeng Rao, On the Elliptic Equations With the First Eigenvalue,Involving the Critial Sobolev Exponents (In Chinese). Advances in Mathematics (China),2004(06):703-711

[45] Ruofeng Rao, EXISTENCE OF MULTIPLE NONTRIVIAL SOLUTIONS FOR THE CRITICAL ELLIPTIC EQUATION $-\Delta u=\lambda_{k} u+$ $|u|^{2^{*}-2} u+f(x, u)$ (In Chinese). Chinese Annals of Mathematics, Series A, 2005(06):749-754

[46] Yi-min MENG, Li-hong HUANG, Zhao-hui YUAN. Exponential Stability Analysis of Cohen-Grossberg Neural Networks with Timevarying Delays. Acta Mathematicae Applicatae Sinica(English Series),2012,28(01):181-192.

[47] Ruofeng Rao, Shouming Zhong, Zhilin Pu. Fixed point and p-stability of T-S fuzzy impulsive reaction-diffusion dynamic neural networks with distributed delay via Laplacian semigroup. Neurocomputing, Volume 33528 March 2019Pages 170-184

[48] T. Wang, L. Xie, and C. E. de Souza, Robust control of a class of uncertain nonlinear systems, Syst. Control Lett., vol. 19, no. 2, pp. 139-149, 1992.

[49] Ruofeng Rao, Qinggao He. Non-zero Solution for the Quasi-linear Elliptic Equation. Chinese Quarterly Journal of Mathematics,2009,24(01), 117-124.

[50] Ruofeng Rao. On the Elliptic Equations With the First Eigenvalue,Involving the Critial Sobolev Exponents (In Chinese). Advances in Mathematics (China), 2004, 33(6),703-711.

[51] Daoyi Xu, Zhiguo Yang, Yumei Huang. Existence-uniqueness and continuation theorems for stochastic functional differential equations. J. Differential Equations, 245 (2008) 1681-1703. 